\title{
REGULARITY ANALYSIS FOR AN ABSTRACT THERMOELASTIC SYSTEM WITH INERTIAL TERM
}

\author{
Zhaobin Kuang ${ }^{1}$, Zhuangyi Liu ${ }^{2,3, *}$ and Hugo D. Fernández Sare ${ }^{4}$
}

\begin{abstract}
In this paper, we provide a complete regularity analysis for the following abstract thermoelastic system with inertial term

$$
\left\{\begin{aligned}
\rho u_{t t}+l A^{\gamma} u_{t t}+\sigma A u-m A^{\alpha} \theta & =0, \\
c \theta_{t}+m A^{\alpha} u_{t}+k A^{\beta} \theta & =0, \\
u(0)=u_{0}, \quad u_{t}(0)=v_{0}, & \theta(0)=\theta_{0},
\end{aligned}\right.
$$

where $A$ is a self-adjoint, positive definite operator on a complex Hilbert space $H$ and

$$
(\alpha, \beta, \gamma) \in E=\left[0, \frac{\beta+1}{2}\right] \times[0,1] \times[0,1]
$$

It is regarded as the second part of Fernández Sare et al. [J. Diff. Eqs. 267 (2019) 7085-7134]. where the asymptotic stability of this model was investigated. We are able to decompose the region $E$ into three parts where the associated semigroups are analytic, of Gevrey classes of specific order, and nonsmoothing, respectively. Moreover, by a detailed spectral analysis, we will show that the orders of Gevrey class are sharp, under proper conditions. We also show that the orders of polynomial stability obtained in Fernández Sare et al. [J. Diff. Eqs. 267 (2019) 7085-7134] are optimal.
\end{abstract}

Mathematics Subject Classification. 35B65, 35K90, 35L90, 47D03, 93D05.

Received June 3, 2020. Accepted November 5, 2020.

\section{INTRODUCTION}

Let $H$ be a complex Hilbert space with the inner product $\langle\cdot, \cdot\rangle$ and the induced norm $\|\cdot\|$. We consider the following abstract system of coupled hyperbolic and parabolic equations:

Keywords and phrases: Hyperbolic-parabolic equations, analytic semigroup, Gevrey class semigroup, polynomial stability.

1 Computer Science Department, Stanford University, Stanford, CA 94305, USA.

2 Department of Mathematics and Statistics, University of Minnesota, Duluth, MN 55812-2496, USA.

3 School of Mathematics, Beijing Institute of Technology, P.R. China.

4 Department of Mathematics, Federal University of Juiz de Fora, CEP 36036-900, Juiz de Fora, MG, Brazil.

* Corresponding author: zliu@d.umn.edu 


$$
\left\{\begin{aligned}
\rho u_{t t}+l A^{\gamma} u_{t t}+\sigma A u-m A^{\alpha} \theta & =0 \\
c \theta_{t}+m A^{\alpha} u_{t}+k A^{\beta} \theta & =0 \\
u(0)=u_{0}, \quad u_{t}(0)=v_{0}, & \theta(0)=\theta_{0}
\end{aligned}\right.
$$

where $A$ is a self-adjoint, positive definite (unbounded) operator on the complex Hilbert space $H, m \neq 0, k>0$ and

$$
(\alpha, \beta, \gamma) \in E=\left[0, \frac{\beta+1}{2}\right] \times[0,1] \times[0,1] .
$$

Our main interest is the regularity of the solution to this system in terms of the parameters $\alpha, \beta, \gamma$. For this purpose, we reformulate system (1.1) in a semigroup setting on the state space

$$
\mathcal{H}=\mathcal{D}\left(A^{\frac{1}{2}}\right) \times \mathcal{D}\left(A^{\frac{\gamma}{2}}\right) \times H,
$$

where any element in $\mathcal{H}$ is denoted by $U=(u, v, w)^{T}$. The inner product in $\mathcal{H}$ is defined by

$$
\left\langle U_{1}, U_{2}\right\rangle_{\mathcal{H}}=\sigma\left\langle A^{\frac{1}{2}} u_{1}, A^{\frac{1}{2}} u_{2}\right\rangle+\rho\left\langle v_{1}, v_{2}\right\rangle+l\left\langle A^{\frac{\gamma}{2}} v_{1}, A^{\frac{\gamma}{2}} v_{2}\right\rangle+c\left\langle\theta_{1}, \theta_{2}\right\rangle,
$$

for all $U_{i}=\left(u_{i}, v_{i}, \theta_{i}\right)^{T} \in \mathcal{H}, i=1,2$, under which $\mathcal{H}$ is a Hilbert space. By denoting $v=u_{t}$ and $U_{0}=$ $\left(u_{0}, v_{0}, w_{0}\right)^{T}$, system (1.1) can be written as an abstract linear evolution equation on the space $\mathcal{H}$,

$$
\left\{\begin{array}{l}
\frac{\mathrm{d} U}{\mathrm{~d} t}(t)=\mathcal{A}_{\alpha, \beta, \gamma} U(t), \quad t \geq 0 \\
U(0)=U_{0}
\end{array}\right.
$$

where the operator $\mathcal{A}_{\alpha, \beta, \gamma}: \mathcal{D}\left(\mathcal{A}_{\alpha, \beta, \gamma}\right) \subseteq \mathcal{H} \rightarrow \mathcal{H}$ is defined for $\alpha \leq \frac{1}{2}$ by

$$
\mathcal{A}_{\alpha, \beta, \gamma}\left[\begin{array}{c}
u \\
v \\
\theta
\end{array}\right]=\left[\begin{array}{c}
v \\
-\left(I+\frac{l}{\rho} A^{\gamma}\right)^{-1} A^{\alpha}\left(\frac{\sigma}{\rho} A^{1-\alpha} u-\frac{m}{\rho} \theta\right) \\
-\frac{m}{c} A^{\alpha} v-\frac{k}{c} A^{\beta} \theta
\end{array}\right]
$$

with domain

$$
\begin{gathered}
D\left(\mathcal{A}_{\alpha, \beta, \gamma}\right)=\left\{(u, v, \theta)^{T} \in \mathcal{H} \mid v \in D\left(A^{1 / 2}\right), \theta \in D\left(A^{\beta}\right), u \in D\left(A^{1-\alpha}\right),\right. \\
\left.\sigma A^{1-\alpha} u-m \theta \in D\left(A^{\alpha-\gamma / 2}\right) \in H\right\} .
\end{gathered}
$$

For $\alpha>\frac{1}{2}$ it is defined by

$$
\mathcal{A}_{\alpha, \beta, \gamma}\left[\begin{array}{c}
u \\
v \\
\theta
\end{array}\right]=\left[\begin{array}{c}
v \\
-\left(I+\frac{l}{\rho} A^{\gamma}\right)^{-1} A^{\alpha}\left(\frac{\sigma}{\rho} A^{1-\alpha} u-\frac{m}{\rho} \theta\right) \\
-A^{\alpha-\frac{1}{2}}\left(\frac{m}{c} A^{\frac{1}{2}} v+\frac{k}{c} A^{\beta-\left(\alpha-\frac{1}{2}\right)} \theta\right)
\end{array}\right]
$$


with domain

$$
\begin{aligned}
& D\left(\mathcal{A}_{\alpha, \beta, \gamma}\right)=\left\{(u, v, \theta)^{T} \in \mathcal{H} \mid v \in D\left(A^{1 / 2}\right), \sigma A^{1-\alpha} u-m \theta \in D\left(A^{\alpha-\gamma / 2}\right),\right. \\
&\left.A^{\beta-(\alpha-1 / 2)} \theta \in H, A^{\alpha-1 / 2}\left[m A^{1 / 2} v+k A^{\beta-(\alpha-1 / 2)} \theta\right] \in H\right\} .
\end{aligned}
$$

It is known that $\mathcal{A}_{\alpha, \beta, \gamma}$ generates a $C_{0}$-semigroup $e^{\mathcal{A}_{\alpha, \beta, \gamma} t}$ of contractions on $\mathcal{H}$, see [8]. Then the solution to the evolution equation (1.2) admits the following representation:

$$
U(t)=e^{\mathcal{A}_{\alpha, \beta, \gamma} t} U_{0}, \quad t \geq 0,
$$

which leads to the well-posedness of (1.2). With this in hand, in general, regularity and stability are the main properties that attract the researchers' attention $[3,4,13,18]$ where these properties are associated with regularity/stability results of their corresponding $C_{0}$ semigroups. Then, before going further, let us recall the definitions of these properties.

Definition 1.1. Let $e^{\mathcal{A} t}$ be a $C_{0}$-semigroup on a Hilbert space $\mathcal{H}$.

1. The semigroup $e^{\mathcal{A} t}$ is said to be analytic if there exists an extension $T(\tau)$ of $e^{\mathcal{A} t}$ to the following set

$$
\Sigma_{\theta} \equiv\{\tau \in \mathbb{C}|| \arg \tau \mid<\theta\} \cup\{0\},
$$

for some $\theta \in\left(0, \frac{\pi}{2}\right)$ such that, for any $x \in \mathcal{H}, \tau \mapsto T(\tau) x$ is continuous on $\Sigma_{\theta}$ satisfying the following semigroup property

$$
T\left(\tau_{1}+\tau_{2}\right)=T\left(\tau_{1}\right) T\left(\tau_{2}\right), \quad \forall \tau_{1}, \tau_{2} \in \Sigma_{\theta}, \text { with } \tau_{1}+\tau_{2} \in \Sigma_{\theta},
$$

and $\tau \mapsto T(\tau)$ is analytic over $\Sigma_{\theta} \backslash\{0\}$ in the uniform operator topology of $\mathcal{L}(\mathcal{H})$ (the space of all linear bounded operators from $\mathcal{H}$ to $\mathcal{H})$.

2. Semigroup $e^{\mathcal{A t}}$ is said to be of Gevrey class $\delta$ (with $\delta>1$ ) if it is infinitely differentiable and, for any compact set $\mathcal{K} \subset(0, \infty)$ and any $\theta>0$, there exists a constant $K=K(\theta, \mathcal{K})$, such that

$$
\left\|\mathcal{A}^{n} e^{\mathcal{A} t}\right\|_{\mathcal{L}(\mathcal{H})} \leq K \theta^{n}(n !)^{\delta}, \quad \forall t \in \mathcal{K}, n \geq 0
$$

3. Semigroup $e^{\mathcal{A} t}$ is said to be differentiable if, for any $x \in \mathcal{H}, t \mapsto e^{\mathcal{A} t} x$ is differentiable on $(0, \infty)$.

4. Semigroup $e^{\mathcal{A} t}$ is said to be exponentially stable with decay rate $\omega>0$ if there exists a constant $M \geq 1$ such that

$$
\left\|e^{\mathcal{A} t}\right\| \leq M e^{-\omega t}, \quad t \geq 0
$$

5. Semigroup $e^{\mathcal{A} t}$ is said to be polynomially stable of order $j>0$ if there exists a constant $M>0$ such that

$$
\left\|e^{\mathcal{A} t} \mathcal{A}^{-1}\right\| \leq M t^{-j}, \quad t>0 .
$$

Note that, in Definition 1.1, the first three items are about the regularity of $C_{0}$-semigroups $([5,7,14,17,19])$ and the last two are about the asymptotic stability of $C_{0}$-semigroups $([6,7,17])$. Also note that, if $\delta=1$ in (1.5), the associated semigroup is analytic; see [19].

Returning to system (1.1), the case of $\gamma=0$ was first considered in [1] for exponential stability. Then the region of $C^{\infty}$ smoothness was founded in [16]. A complete picture of the stability and regularity of this model were given in $[9,10]$. In the case $\gamma>0$, the analysis become more demanding due to the following challenges: How to divide the parameter region $E$ into subregions with specific stability and regularity properties due to 


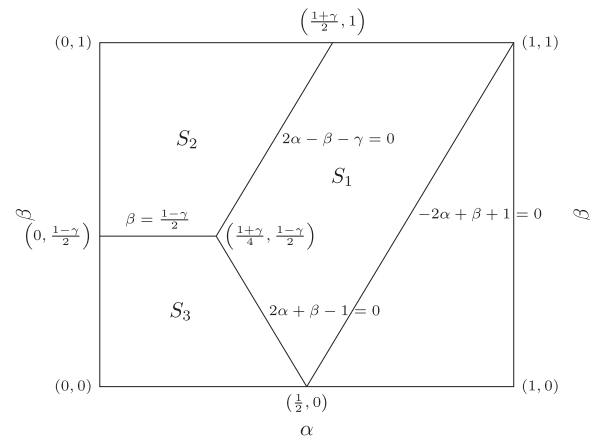

(a) Region's boundaries.

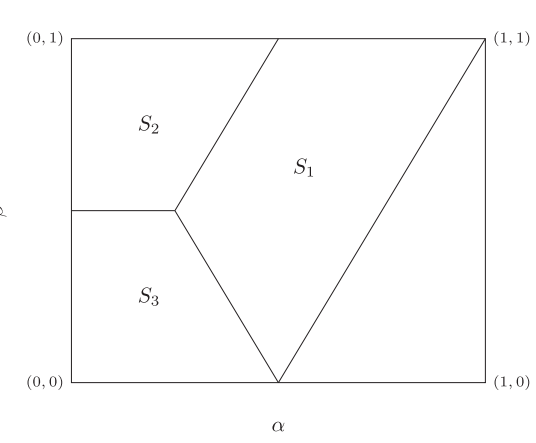

(b) $\gamma=0$

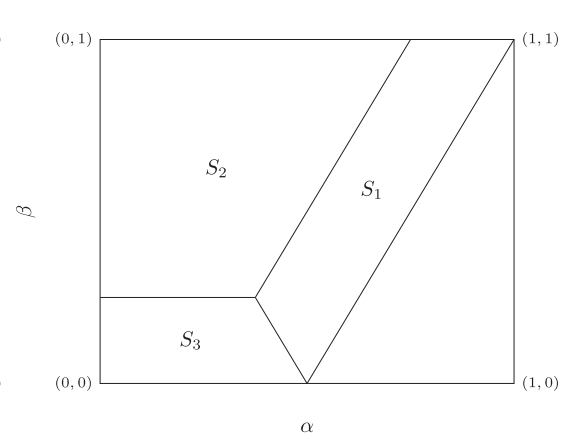

(c) $\gamma=\frac{1}{2}$

Figure 1. Regions $S_{1}, S_{2}$, and $S_{3}$ for some $\gamma$ 's.

the additional parameter $\gamma$ ? How to determine the order of polynomial stability and Gevrey class in terms of the parameters? How to prove these properties in each subregion? Recently, the stability analysis of the system (1.1) has been reported in [8]. For reader's convenience, we give a brief summary here.

1. The semigroup $e^{\mathcal{A}_{\alpha, \beta, \gamma} t}$ is exponentially stable in $S_{1}$,

2. The semigroup $e^{\mathcal{A}_{\alpha, \beta, \gamma} t}$ is polynomially stable of order $\delta>-\frac{1}{\mu(\alpha, \beta, \gamma)}$ in $S_{2} \cup S_{3}$ with

$$
\mu(\alpha, \beta, \gamma)= \begin{cases}\frac{2 \alpha-\beta-\gamma}{(1-\gamma) / 2}, & (\alpha, \beta, \gamma) \in S_{2} \\ \frac{2 \alpha+\beta-1}{(1-\gamma) / 2}, & (\alpha, \beta, \gamma) \in S_{3}\end{cases}
$$

where

$$
\begin{aligned}
& S_{1}=\{(\alpha, \beta, \gamma) \in E: 1 \leq 2 \alpha+\beta, \quad \gamma \leq 2 \alpha-\beta \leq 1\}, \\
& S_{2}=\left\{(\alpha, \beta, \gamma) \in E: 0 \leq \alpha<\frac{\beta+\gamma}{2}, \frac{1-\gamma}{2} \leq \beta \leq 1\right\}, \\
& S_{3}=\left\{(\alpha, \beta, \gamma) \in E: 0 \leq \alpha<\frac{1-\beta}{2}, 0 \leq \beta<\frac{1-\gamma}{2}\right\} .
\end{aligned}
$$

Examples of regions $S_{1}, S_{2}$, and $S_{3}$ are given in Figure 1.

As mentioned previously, the aim of this paper is on conducting a regularity analysis of system (1.1). We organize the paper as follows. In Section 2, we will summarize the main results of the paper. Specifically,

(a) We will decompose the parameter region $E$ into three parts where the semigroup associated with the system is analytic, of specific order of Gevrey class, and non-smoothing, respectively.

(b) By a detailed spectral analysis, we will show that the orders of Gevrey class is sharp, under proper conditions.

(c) We also show that the orders of polynomial stability obtained in [8] are optimal.

In Section 3, we provide a list frequency domain characterization of semigroup properties, useful to proof our results. Section 4 is devoted to the Proof of main result (a). Finally, proofs of results (b) and (c) are provided in Section 5 and Section 6. 


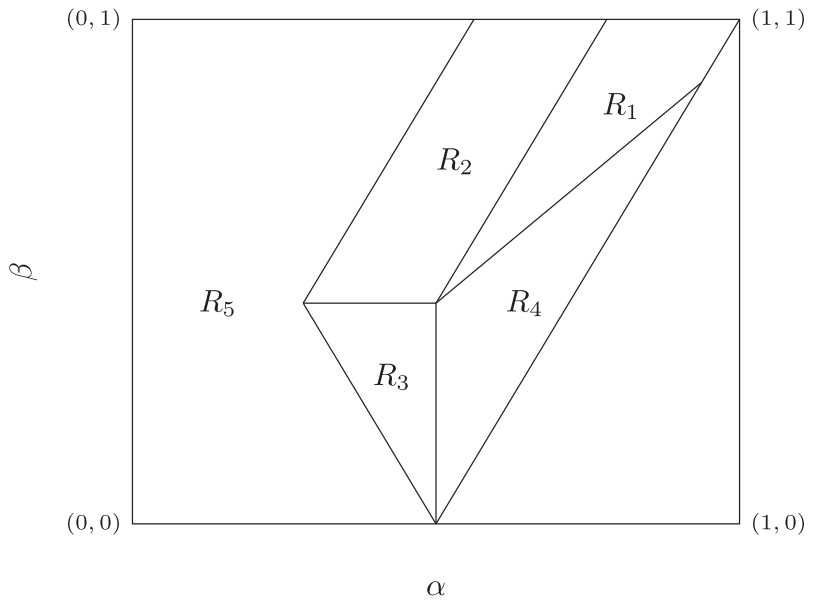

(a) IDs and locations of various regions.

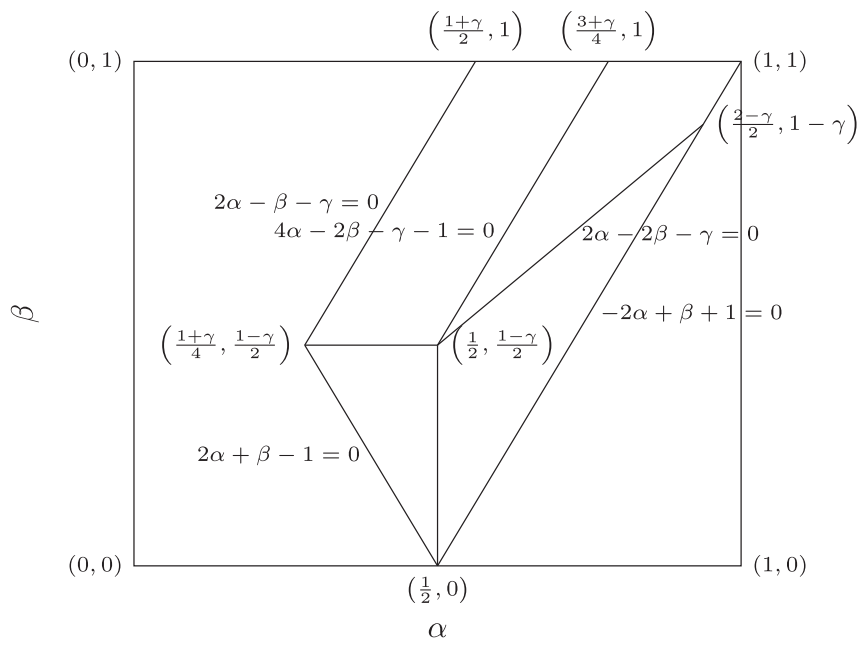

(b) boundaries of various regions.

FiguRE 2. Regions given in (2.1). The figures are plotted choosing $\gamma=\frac{1}{8}$.

We would like to remark here that the method we introduce in Section 5-6 to analyze the asymptotic behavior of the eigenvalues of the system, which is a cubic equation with coefficients depending on three parameters $\alpha, \beta, \gamma$ in region $E$, is crucial to our success.

Before ending this section, we give an example to illustrate the motivation of our investigation. Example: The thermoelastic plate equation

$$
\left\{\begin{array}{l}
u_{t t}(x, t)-l \Delta u_{t t}(x, t)=-E \Delta^{2}(x, t)+k \Delta \theta(x, t), \quad \text { in } \Omega \times(0, \infty), \\
c \theta_{t}(x, t)=-\Delta u_{t}(x, t)+k \Delta \theta(x, t), \quad \text { in } \Omega \times(0, \infty), \\
u(x, t)=\Delta u(x, t)=\theta(x, t)=0, \quad \text { on } \partial \Omega \times(0, \infty), \\
u(x, 0)=u_{0}(x), \quad \nabla u(x, 0)=u_{1}(x), \quad \theta(x, 0)=\theta_{0}(x) \quad \text { in } \Omega .
\end{array}\right.
$$

It is known that the semigroup associated with this system is analytic when $l=0$, but is not analytic when $l>0$, see for example [11-13]. This system falls into the framework of system (1.1) for $A=-\Delta,(\alpha, \beta, \gamma)=$ $\left(\frac{1}{2}, \frac{1}{2}, 0\right.$ or 1$)$.

For the sake of simplicity and without loss of generality, in our analysis we assume $l=\rho=\sigma=m=c=k=1$.

\section{MAIN RESULTS}

To state the main results, we first divide the parameter region $E$ into the following subregions:

$$
\left\{\begin{array}{l}
R_{1}=\{(\alpha, \beta, \gamma) \mid \beta \leq 1,-2 \alpha+\beta+1>0,2 \alpha-2 \beta-\gamma \leq 0,4 \alpha-2 \beta-\gamma-1 \geq 0,0 \leq \gamma \leq 1\}, \\
R_{2}=\left\{(\alpha, \beta, \gamma) \mid \frac{1-\gamma}{2} \leq \beta \leq 1,4 \alpha-2 \beta-\gamma-1<0,2 \alpha-\beta-\gamma>0,0 \leq \gamma<1\right\}, \\
R_{3}=\left\{(\alpha, \beta, \gamma) \mid \alpha<\frac{1}{2}, \beta<\frac{1-\gamma}{2}, 2 \alpha+\beta-1>0,0 \leq \gamma<1\right\}, \\
R_{4}=\left\{(\alpha, \beta, \gamma) \mid \alpha \geq \frac{1}{2}, 2 \alpha-2 \beta-\gamma>0,-2 \alpha+\beta+1>0,0 \leq \gamma<1\right\}, \\
R_{5}=E \backslash\left(R_{1} \cup R_{2} \cup R_{3} \cup R_{4}\right) .
\end{array}\right.
$$

The regions defined in (2.1) are visualized in Figure 2. Additionally, Figure 3 shows how these regions change at various $\gamma$ 's. 


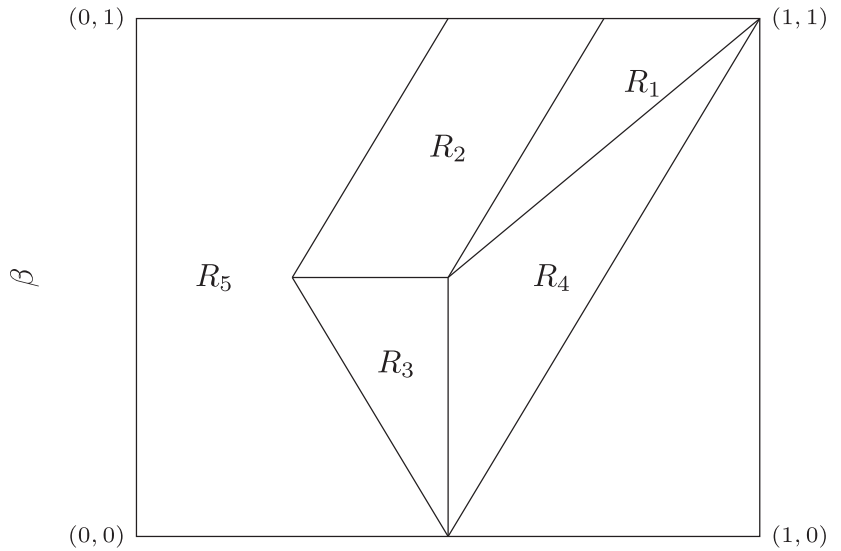

$\alpha$

(a) $\gamma=0$

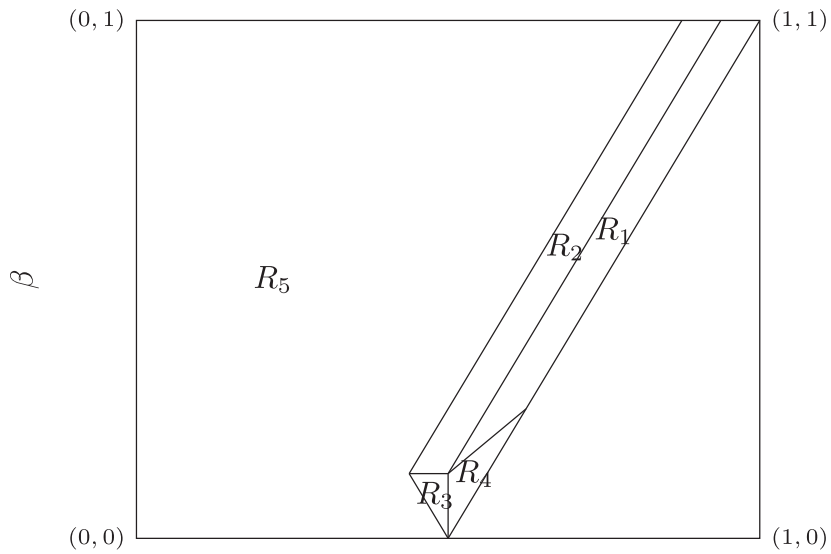

$\alpha$

(c) $\gamma=\frac{3}{4}$

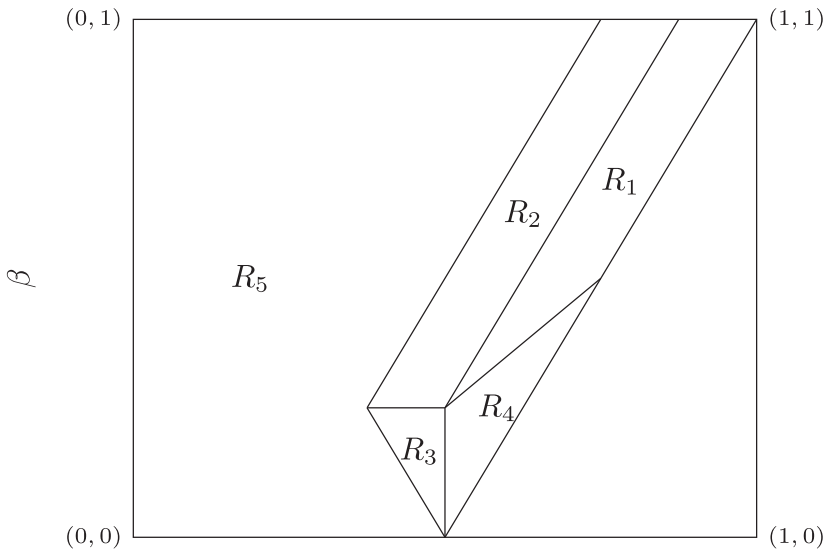

$\alpha$

(b) $\gamma=\frac{1}{2}$

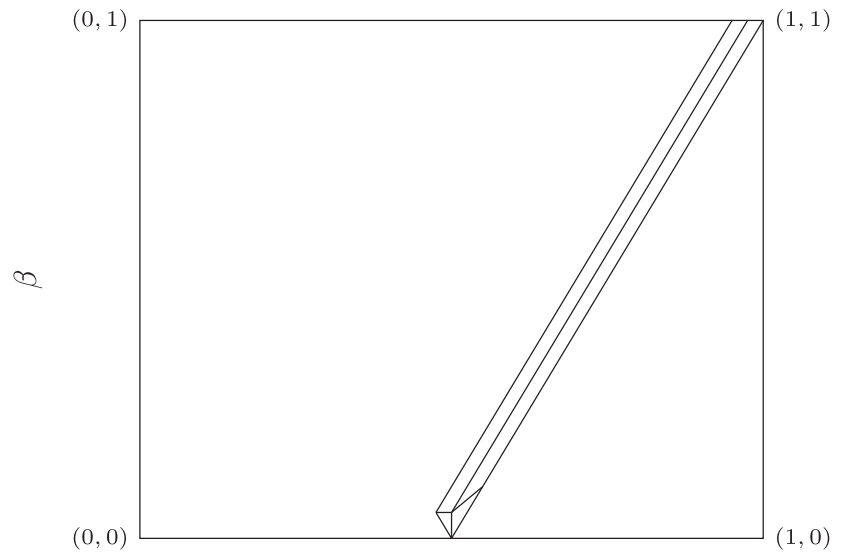

$\alpha$

(d) $\gamma=\frac{9}{10}$

FiguRE 3. Regions defined by (2.1) at various $\gamma$ 's.

With the regions defined above, we can decompose $E$ into three parts where the semigroup associated with (1.1) is analytic, of specific order of Gevrey classes, and non-smoothing, respectively via Theorem 2.1.

Theorem 2.1. The semigroup $e^{\mathcal{A}_{\alpha, \beta, \gamma} t}$ has the following regularity properties:

(i) It is analytic in $R_{1}$;

(ii) It is of Gevrey class $\delta>\frac{1}{\mu(\alpha, \beta, \gamma)}$ in $R_{2} \cup R_{3} \cup R_{4}$ with

$$
\mu(\alpha, \beta, \gamma)= \begin{cases}\frac{2 \alpha-\beta-\gamma}{(1-\gamma) / 2}, & (\alpha, \beta, \gamma) \in R_{2} \\ \frac{2 \alpha+\beta-1}{(1-\gamma) / 2}, & (\alpha, \beta, \gamma) \in R_{3} \\ \frac{\beta}{\alpha-\gamma / 2}, & (\alpha, \beta, \gamma) \in R_{4}\end{cases}
$$

(iii) It is not differentiable in $R_{5}$. 
It should be noticed that when $\gamma=1$, the analytic region $R_{1}$ degenerates to the line $-2 \alpha+\beta+1=0$. Furthermore, the sharpness of the orders of Gevrey class corresponding to (2.2) of Theorem 2.1 as well as the sharpness of the orders of polynomial stability corresponding to (1.6) can be characterized by Theorem 2.2:

Theorem 2.2. If $A$ admits a sequence of eigenvalues $\mu_{n} \in \mathbb{R}$ such that $\lim _{n \rightarrow \infty} \mu_{n}=\infty$, then the Gevrey class orders in (2.2) of Theorem 2.1 are sharp in the following sense: For any $\varepsilon>0$, the semigroup is not Gevrey class of order $\frac{1}{\mu(\alpha, \beta, \gamma)+\varepsilon}$. Furthermore, the orders of polynomial stability in (1.6) are also sharp: for any $\varepsilon>0$, the semigroup is not polynomially stable of order $-\frac{1}{\mu(\alpha, \beta, \gamma)+\varepsilon}$.

The significance of the above results includes the following:

- System (1.1) covers a variety of partial differential equations. Within this framework, the stability and regularity problem of the system are now completely resolved. It is possible to extend the results to the case when operators in the system are not in the form of fractional power of $A$, but equivalent to it in some sense. See [14] for a simpler system.

- The semigroup properties obtained in [8] and this paper provides important information for the study of nonlinear evolution equations related to the linear system (1.1).

- The asymptotic analysis of eigenvalues for system (1.1) is crucial to precisely divide the parameter region $E$ into subregions corresponding to the semigroup properties. The method designed presented in Section 5-6 provides a powerful mean to fulfill this formidable task, which can be applied to other type of general systems with parameters.

\section{Frequency domain Characterization of SEMigroup's PROPERTIES}

In this section, we will present the frequency domain characterization of semigroup properties, which are our tools of proving the regularity results in Theorem 2.1.

Lemma 3.1. Let $\mathcal{A}: \mathcal{D}(\mathcal{A}) \subseteq \mathcal{H} \rightarrow \mathcal{H}$ generate a $C_{0}$-semigroup $e^{\mathcal{A} t}$ on $\mathcal{H}$ such that

$$
\left\|e^{\mathcal{A} t}\right\| \leq M, \quad \forall t \geq 0
$$

for some $M \geq 1$ and

$$
i \lambda \in \rho(\mathcal{A}), \quad \forall \lambda \in \mathbb{R},|\lambda| \text { large enough. }
$$

Then the following hold:

1. Semigroup $e^{\mathcal{A} t}$ is analytic if and only if for some $a \in \mathbb{R}$ and $b, C>0$ such that

$$
\rho(\mathcal{A}) \supseteq \Sigma(a, b) \equiv\{\lambda \in \mathbb{C}|\operatorname{Re} \lambda>a-b| \operatorname{Im} \lambda \mid\},
$$

and

$$
\left\|(i \lambda-\mathcal{A})^{-1}\right\| \leq \frac{C}{1+|\lambda|}, \quad \lambda \in \Sigma(a, b)
$$

This is the case if and only if

$$
\varlimsup_{\lambda \in \mathbb{R},|\lambda| \rightarrow \infty}|\lambda|\left\|(i \lambda-\mathcal{A})^{-1}\right\|<\infty .
$$


2. Semigroup $e^{\mathcal{A} t}$ is of Gevrey class $\delta>1$ if and only if for any $b, \tau>0$, there are constants $a \in \mathbb{R}$ and $C>0$ depending on $b, \tau, \delta$ such that

$$
\rho(\mathcal{A}) \supseteq \Sigma_{b}(\delta) \equiv\left\{\left.\lambda \in \mathbb{C}|\operatorname{Re} \lambda>a-b| \operatorname{Im} \lambda\right|^{\frac{1}{\delta}}\right\}
$$

and

$$
\left\|(i \lambda-\mathcal{A})^{-1}\right\| \leq C\left(e^{-\tau \operatorname{Re} \lambda}+1\right), \quad \forall \lambda \in \Sigma_{b}(\delta) .
$$

This is the case, in particular, if for some $\mu \in\left(\delta^{-1}, 1\right)$,

$$
\varlimsup_{\lambda \in \mathbb{R},|\lambda| \rightarrow \infty}|\lambda|^{\mu}\left\|(i \lambda-\mathcal{A})^{-1}\right\|<\infty .
$$

3. Semigroup $e^{\mathcal{A} t}$ is differentiable if and only if for any $b>0$, there are constants $a_{b} \in \mathbb{R}$ and $C_{b}>0$ such that

$$
\rho(\mathcal{A}) \supseteq \Sigma_{b} \equiv\left\{\lambda \in \mathbb{C}\left|\operatorname{Re} \lambda>a_{b}-b \log \right| \operatorname{Im} \lambda \mid\right\}
$$

and

$$
\left\|(i \lambda-\mathcal{A})^{-1}\right\| \leq C_{b}|\operatorname{Im} \lambda|, \quad \forall \lambda \in \Sigma_{b}, \operatorname{Re} \lambda \leq 0 .
$$

This is the case, in particular, if

$$
\varlimsup_{\lambda \in \mathbb{R},|\lambda| \rightarrow \infty} \log |\lambda|\left\|(i \lambda-\mathcal{A})^{-1}\right\|=0 .
$$

4. Semigroup $e^{\mathcal{A} t}$ is exponentially stable if and only if

$$
i \lambda \in \rho(\mathcal{A}), \quad \forall \lambda \in \mathbb{R},
$$

and

$$
\varlimsup_{\lambda \in \mathbb{R},|\lambda| \rightarrow \infty}\left\|(i \lambda-\mathcal{A})^{-1}\right\|<\infty
$$

5. Semigroup $e^{\mathcal{A} t}$ is polynomially stable of order $j>0$ if and only if (3.5) holds and

$$
\varlimsup_{\lambda \in \mathbb{R},|\lambda| \rightarrow \infty}|\lambda|^{-\frac{1}{j}}\left\|(i \lambda-\mathcal{A})^{-1}\right\|<\infty .
$$

For notational simplicity, hereafter, we write $i \lambda-\mathcal{A}$ instead of $i \lambda I-\mathcal{A}$, omitting $I$. In the above result, the regularity and stability properties of the semigroup $e^{\mathcal{A} t}$ are deliberately related to the spectral/resolvent of the generator $\mathcal{A}$. Practically, we will use the limit relations (3.5), (3.8) and (3.11) to establish the regularity property of the semigroup, and use the spectrum relations (3.3), (3.6) and (3.9) to show that the relevant indices are sharp. The following corollary will be useful below. See [10]. 


\section{Corollary 3.2.}

1. Suppose $\sigma(\mathcal{A})$ contains a sequence $\lambda_{n}$ such that

$$
\lim _{n \rightarrow \infty} \operatorname{Re} \lambda_{n}=a, \quad \lim _{n \rightarrow \infty}\left|\lambda_{n}\right|=\infty
$$

for some $a \in \mathbb{R}$, then the semigroup $e^{\mathcal{A} t}$ is not differentiable.

2. Suppose there exists a sequence $\lambda_{n} \in \sigma(\mathcal{A})$ such that

$$
\varlimsup_{n \rightarrow \infty} \frac{\operatorname{Re} \lambda_{n}}{\left|\operatorname{Im} \lambda_{n}\right|^{\frac{1}{\delta}}}=0
$$

then $e^{\mathcal{A} t}$ is not of Gevrey class $\delta$.

\section{Proof of Theorem 2.1}

In this section, we will prove Theorem 2.1. It is technical and lengthy. Let us now make some preparations. First of all, in our proof, the following interpolation theorem will play a crucial role.

Lemma 4.1. Let $A: \mathcal{D}(A) \subseteq H$ be self-adjoint and positive definite. Then

$$
\left\|A^{p} x\right\| \leq\left\|A^{q} x\right\|^{\frac{p-r}{q-r}}\left\|A^{r} x\right\|^{\frac{q-p}{q-r}}, \quad \forall 0 \leq r \leq p \leq q, x \in \mathcal{D}\left(A^{q}\right) .
$$

In particular, for any $\theta \in\left[0, \frac{1}{2}\right]$, one has (with $r=0, p=\theta$, and $q=\frac{1}{2}$ )

$$
\left\|A^{\theta} x\right\| \leq\left\|A^{\frac{1}{2}} x\right\|^{2 \theta}\|x\|^{1-2 \theta}, \quad \forall x \in \mathcal{D}\left(A^{\frac{1}{2}}\right),
$$

and for any $\theta \in\left[\frac{1}{2}, 1\right]$ (with $r=\frac{1}{2}, p=\theta$, and $q=1$ )

$$
\left\|A^{\theta} x\right\| \leq\|A x\|^{2 \theta-1}\left\|A^{\frac{1}{2}} x\right\|^{2(1-\theta)}, \quad \forall x \in \mathcal{D}(A) .
$$

Next, for any $\lambda \in \mathbb{R}$, and any $U \equiv(u, v, w)^{T} \in \mathcal{D}\left(\mathcal{A}_{\alpha, \beta, \gamma}\right)$,

$$
\left(i \lambda-\mathcal{A}_{\alpha, \beta, \gamma}\right) U=\left(\begin{array}{c}
i \lambda u-v \\
i \lambda v+\left(I+A^{\gamma}\right)^{-1} A^{\alpha}\left(A^{1-\alpha} u_{n}-\theta_{n}\right) \\
i \lambda \theta+T\left(v_{n}, \theta_{n}\right)
\end{array}\right),
$$

where

$$
T\left(v_{n}, \theta_{n}\right):= \begin{cases}A^{\alpha} v_{n}+A^{\beta} \theta_{n}, & \text { for } \alpha \leq 1 / 2 \\ A^{\alpha-\frac{1}{2}}\left(A^{\frac{1}{2}} v_{n}+A^{\beta-\alpha+\frac{1}{2}} \theta_{n}\right), & \text { for } \alpha>1 / 2 .\end{cases}
$$

Our proof for Theorem 2.1 will be based on contradiction arguments. Suppose for some given $(\alpha, \beta, \gamma) \in S$ and $\mu \in[0,1]$, without having any specific relations among them, that the following is not true:

$$
\varlimsup_{\lambda \in \mathbb{R},|\lambda| \rightarrow \infty}|\lambda|^{\mu}\left\|\left(i \lambda-\mathcal{A}_{\alpha, \beta, \gamma}\right)^{-1}\right\|<\infty .
$$


Then there exists a sequence $\left\{\left(\lambda_{n}, U_{n}\right) \mid n \geq 1\right\} \subseteq \mathbb{R} \times \mathcal{D}\left(\mathcal{A}_{\alpha, \beta, \gamma}\right)$ with $U_{n} \equiv\left(u_{n}, v_{n}, \theta_{n}\right)^{T}$, and

$$
\left\{\begin{array}{l}
\lim _{n \rightarrow \infty}\left|\lambda_{n}\right|=\infty \\
\left\|U_{n}\right\|_{\mathcal{H}}^{2}=\left\|A^{\frac{1}{2}} u_{n}\right\|^{2}+\left\|v_{n}\right\|^{2}+\left\|A^{\frac{\gamma}{2}} v_{n}\right\|^{2}+\left\|\theta_{n}\right\|^{2}=1, \quad n \geq 1
\end{array}\right.
$$

such that

$$
\lim _{n \rightarrow \infty}\left|\lambda_{n}\right|^{-\mu}\left\|\left(i \lambda_{n}-\mathcal{A}_{\alpha, \beta, \gamma}\right) U_{n}\right\|_{\mathcal{H}}=0
$$

i.e. (note (4.4)), without loss of generality, $\lambda_{n}>0$,

$$
\begin{aligned}
i \lambda_{n}^{1-\mu} A^{\frac{1}{2}} u_{n}-\lambda_{n}^{-\mu} A^{\frac{1}{2}} v_{n}=o(1), \quad \text { in } \quad H, & \\
i \lambda_{n}^{1-\mu}\left(I+A^{\gamma}\right) v_{n}+\lambda_{n}^{-\mu} A^{\alpha}\left(A^{1-\alpha} u_{n}-\theta_{n}\right) & \longrightarrow 0, \quad \text { in } \mathcal{D}\left(A^{\frac{\gamma}{2}}\right)^{\prime}, \\
i \lambda_{n}^{1-\mu} \theta_{n}+\lambda_{n}^{-\mu} T\left(v_{n}, \theta_{n}\right)=o(1) & \text { in } \quad H,
\end{aligned}
$$

where $(4.8 \mathrm{~b})$ is derived by acting $\left(I+A^{\gamma}\right)$ on the second component of (4.4). Hereafter, if not specified, $o(1)$ stands for a vector in $H$ (or a quantity in $\mathbb{R}$ ) which goes to zero as $n \rightarrow \infty$, and $O(1)$ a bounded vector in $H$ (or uniformly in $n \geq 1$, in $\mathbb{R}$ ). The advantage of using such a notation is that (4.8a)-(4.8c) can be regarded as a system of equations, which will be convenient to the analysis later on. Furthermore, acting $A^{-\frac{\gamma}{2}}$ on $(4.8 \mathrm{~b})$ yields,

$$
i \lambda_{n}^{1-\mu}\left(A^{-\frac{\gamma}{2}}+A^{\frac{\gamma}{2}}\right) v_{n}+\lambda_{n}^{-\mu} A^{\alpha-\frac{\gamma}{2}}\left(A^{1-\alpha} u_{n}-\theta_{n}\right)=o(1)
$$

where the l.h.s. of $(4.9) \in H$. For the sequence $\left\{\left(\lambda_{n}, u_{n}, v_{n}, \theta_{n}\right)\right\}$ satisfying (4.8a)-(4.8c), we have the following result.

Lemma 4.2. For the sequence $\left\{\left(\lambda_{n}, u_{n}, v_{n}, \theta_{n}\right)\right\}$ satisfying (4.6)-(4.7), the following convergences are true:

$$
\begin{gathered}
\lambda_{n}^{-\frac{\mu}{2}} A^{\frac{\beta}{2}} \theta_{n}=o(1), \\
i\left\|v_{n}\right\|^{2}+i\left\|A^{\frac{\gamma}{2}} v_{n}\right\|^{2}-i\left\|A^{\frac{1}{2}} u_{n}\right\|^{2}-\left\langle\lambda_{n}^{-1+\frac{\mu}{2}} A^{\frac{\beta}{2}} \theta_{n}, \lambda_{n}^{-\frac{\mu}{2}} A^{\alpha-\frac{\beta}{2}} v_{n}\right\rangle=o(1) . \\
\lambda_{n}^{-1} A^{\alpha-\frac{\gamma}{2}}\left(A^{1-\alpha} u_{n}-\theta_{n}\right)=O(1) . \\
i \lambda_{n}^{1-\mu}\left\|\theta_{n}\right\|^{2}+\lambda_{n}^{-\mu}\left\langle A^{\frac{1}{2}} v_{n}, A^{\alpha-\frac{1}{2}} \theta_{n}\right\rangle=o(1) .
\end{gathered}
$$

Proof. To show (4.10a) is true, consider:

$$
\begin{aligned}
& \operatorname{Re}\left\langle\lambda_{n}^{-\mu}\left(i \lambda_{n}-\mathcal{A}_{\alpha, \beta, \gamma}\right) U_{n}, U_{n}\right\rangle_{\mathcal{H}}=-\operatorname{Re}\left\langle\lambda_{n}^{-\mu} \mathcal{A}_{\alpha, \beta, \gamma} U_{n}, U_{n}\right\rangle_{\mathcal{H}} \\
= & -\lambda_{n}^{-\mu} \operatorname{Re}\left(\left\langle A^{\frac{1}{2}} v_{n}, A^{\frac{1}{2}} u_{n}\right\rangle+\left\langle A^{\alpha-\gamma / 2}\left(A^{1-\alpha} u_{n}-\theta_{n}\right), A^{\gamma / 2} v_{n}\right\rangle+\left\langle T\left(v_{n}, \theta_{n}\right), \theta_{n}\right\rangle\right) \\
= & \left\|\lambda_{n}^{-\frac{\mu}{2}} A^{\frac{\beta}{2}} \theta_{n}\right\|^{2} .
\end{aligned}
$$


For the analysis of the last term, see reference [8]. Then, by (4.6) and (4.7), we obtain

$$
\left\|\lambda_{n}^{-\frac{\mu}{2}} A^{\frac{\beta}{2}} \theta_{n}\right\|^{2}=o(1)
$$

To show (4.10b) is true, we see that since $\mu \in[0,1], \lambda_{n}^{\mu-1} A^{\frac{\gamma}{2}} v_{n}$ is bounded in $H$ because of (4.6). Taking the inner product of (4.9) with $\lambda_{n}^{\mu-1} A^{\frac{\gamma}{2}} v_{n}$ in $H$ yields:

$$
\begin{aligned}
& \left\langle i\left(I+A^{\gamma}\right) v_{n}, v_{n}\right\rangle+\left\langle\lambda_{n}^{-1} A^{\alpha-\frac{1}{2}}\left(A^{1-\alpha} u_{n}-\theta_{n}\right), A^{\frac{1}{2}} v_{n}\right\rangle \\
& \quad=i\left\|v_{n}\right\|^{2}+i\left\|A^{\frac{\gamma}{2}} v_{n}\right\|^{2}+\left\langle A^{\frac{1}{2}} u_{n}, \lambda_{n}^{-1} A^{\frac{1}{2}} v_{n}\right\rangle-\lambda_{n}^{-1}\left\langle A^{\alpha-\frac{1}{2}} \theta_{n}, A^{\frac{1}{2}} v_{n}\right\rangle \\
& \quad=i\left\|v_{n}\right\|^{2}+i\left\|A^{\frac{\gamma}{2}} v_{n}\right\|^{2}-i\left\|A^{\frac{1}{2}} u_{n}\right\|^{2}-\left\langle\lambda_{n}^{-1+\frac{\mu}{2}} A^{\frac{\beta}{2}} \theta_{n}, \lambda_{n}^{-\frac{\mu}{2}} A^{\alpha-\frac{\beta}{2}} v_{n}\right\rangle+o(1)=o(1),
\end{aligned}
$$

where $A^{\alpha-\frac{1}{2}} \theta_{n} \in H$ because $\alpha-\frac{1}{2} \leq \frac{\beta}{2}$ in $S_{1}$. Also, the penultimate equality can be obtained by multiplying (4.8a) with the bounded $\lambda_{n}^{\mu-1}$ to yield $i A^{\frac{1}{2}} u_{n}=\lambda_{n}^{-1} A^{\frac{1}{2}} v_{n}+o(1)$. (4.10c) follows from multiplying (4.9) by the bounded $\lambda_{n}^{\mu-1}$ and the fact that $\left\|A^{\frac{\gamma}{2}} v_{n}\right\| \leq 1$. Finally, (4.10d) is obtained from the inner product of (4.8c) with $\theta_{n}$ in $H$ and using (4.10a).

Theorem 4.3. Let

$$
\mu(\alpha, \beta, \gamma)= \begin{cases}1, & (\alpha, \beta, \gamma) \in R_{1} \\ \frac{2(2 \alpha-\beta-\gamma)}{1-\gamma}, & (\alpha, \beta, \gamma) \in R_{2} \\ \frac{2(2 \alpha+\beta-1)}{1-\gamma}, & (\alpha, \beta, \gamma) \in R_{3} \\ \frac{2 \beta}{2 \alpha-\gamma}, & (\alpha, \beta, \gamma) \in R_{4}\end{cases}
$$

where $R_{i}, i=1,2,3,4$ are defined by (2.1). Then

$$
\varlimsup_{\lambda \in \mathbb{R},|\lambda| \rightarrow \infty}|\lambda|^{\mu(\alpha, \beta, \gamma)}\left\|\left(i \lambda-\mathcal{A}_{\alpha, \beta, \gamma}\right)^{-1}\right\|<\infty .
$$

Proof. In order to prove the result, let us argue by contradiction arguments. In fact, assuming that (4.13) is false, then the convergence (4.6)-(4.9) and also Lemma 4.2 hold. We will show that $\left\|U_{n}\right\|_{\mathcal{H}}=o(1)$, which is a contradiction with (4.6). We divide our analysis by cases, depending on the region $R_{i}, i=1,2,3,4$.

Case 1. Let $(\alpha, \beta, \gamma) \in R_{1}$ where

$$
R_{1}=\{(\alpha, \beta, \gamma) \mid \beta \leq 1,-2 \alpha+\beta+1 \geq 0,2 \alpha-2 \beta-\gamma \leq 0,4 \alpha-2 \beta-\gamma-1 \geq 0,0 \leq \gamma \leq 1\},
$$

and is shown in Figure 4 where $\gamma$ is $\frac{1}{4}, \frac{1}{2}$, and $\frac{3}{4}$, respectively.

Take $\mu=1$. The inner product of $(4.8 \mathrm{c})$ with $A^{\alpha-\beta} v_{n}$ in $H$, which is bounded since $\alpha-\beta \leq \frac{\gamma}{2}$ in $R_{1}$ and $\left\|A^{\frac{\gamma}{2}} v_{n}\right\|$ is bounded, gives

$$
\left\langle i \theta_{n}, A^{\alpha-\beta} v_{n}\right\rangle+\left\|\lambda_{n}^{-\frac{1}{2}} A^{\alpha-\frac{\beta}{2}} v_{n}\right\|^{2}+\lambda_{n}^{-1}\left\langle A^{\alpha-\frac{1}{2}} \theta_{n}, A^{\frac{1}{2}} v_{n}\right\rangle=o(1) .
$$

It follows from (4.10d) and $\mu=1$ that $\lambda_{n}^{-1}\left(A^{\alpha-\frac{1}{2}} \theta_{n}, A^{\frac{1}{2}} v_{n}\right)$ is bounded. Therefore, we obtain from (4.14) that

$$
\left\|\lambda_{n}^{-\frac{1}{2}} A^{\alpha-\frac{\beta}{2}} v_{n}\right\|^{2}=O(1)
$$

because $\left\langle i \theta_{n}, A^{\alpha-\beta} v_{n}\right\rangle$ is also bounded. Consequently, (4.15) implies that 


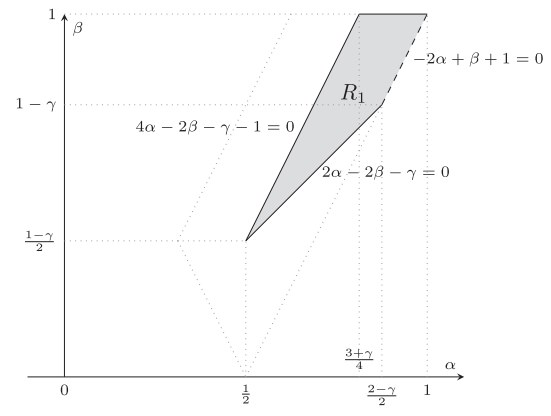

(a) $\gamma=\frac{1}{4}$

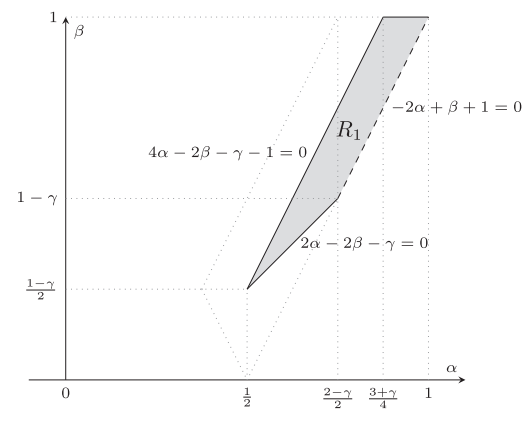

(b) $\gamma=\frac{1}{2}$

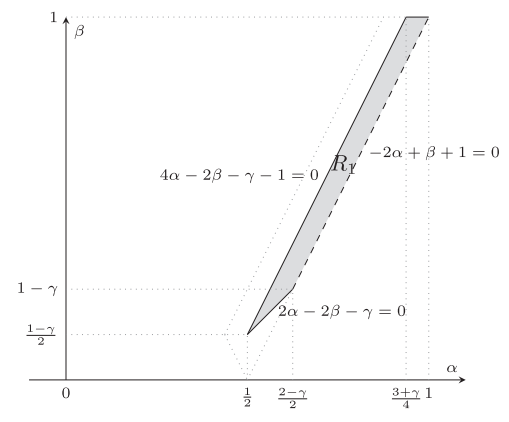

(c) $\gamma=\frac{3}{4}$

Figure 4. Visualization of $R_{1}$ at various $\gamma$ 's.

$$
\left|\lambda_{n}^{-1}\left\langle A^{\alpha-\frac{1}{2}} \theta_{n}, A^{\frac{1}{2}} v_{n}\right\rangle\right|=\left|\left\langle\lambda_{n}^{-\frac{1}{2}} A^{\frac{\beta}{2}} \theta_{n}, \lambda_{n}^{-\frac{1}{2}} A^{\alpha-\frac{\beta}{2}} v_{n}\right\rangle\right| \leq\left\|\lambda_{n}^{-\frac{1}{2}} A^{\frac{\beta}{2}} \theta_{n}\right\|\left\|\lambda_{n}^{-\frac{1}{2}} A^{\alpha-\frac{\beta}{2}} v_{n}\right\|=o(1),
$$

where we have used also (4.10a). Now, returning to (4.10d), the estimate (4.16) implies that

$$
\left\|\theta_{n}\right\|=o(1)
$$

On the other hand, from (4.8c) and (4.17) we have

$$
\lambda_{n}^{-1} A^{\alpha-\frac{1}{2}}\left(A^{\frac{1}{2}} v_{n}+A^{\beta-\alpha+\frac{1}{2}} \theta_{n}\right)=o(1) .
$$

Since $\alpha \geq \frac{1}{2}$ in $R_{1},\left\|A^{1-\alpha} u_{n}\right\|=O(1)$ by (4.6). Then, taking the inner product of (4.18) with $A^{1-\alpha} u_{n}$ in $H$, we obtain

$$
\begin{aligned}
o(1) & =\left\langle\lambda_{n}^{-1} A^{\alpha-\frac{1}{2}}\left(A^{\frac{1}{2}} v_{n}+A^{\beta-\alpha+\frac{1}{2}} \theta_{n}\right), A^{1-\alpha} u_{n}\right\rangle \\
& =\left\langle\lambda_{n}^{-1}\left(A^{\frac{1}{2}} v_{n}+A^{\beta-\alpha+\frac{1}{2}} \theta_{n}\right), A^{\frac{1}{2}} u_{n}\right\rangle \\
& =i\left\|A^{\frac{1}{2}} u_{n}\right\|^{2}+o(1)+\lambda_{n}^{-1}\left\langle A^{\beta-\alpha+\frac{1}{2}} \theta_{n}, A^{\frac{1}{2}} u_{n}-A^{\alpha-\frac{1}{2}} \theta_{n}\right\rangle+\lambda_{n}^{-1}\left\langle A^{\beta-\alpha+\frac{1}{2}} \theta_{n}, A^{\alpha-\frac{1}{2}} \theta_{n}\right\rangle \\
& =-i\left\|A^{\frac{1}{2}} u_{n}\right\|^{2}+o(1)+\left\langle\lambda_{n}^{-\frac{1}{2}} A^{\frac{\beta}{2}} \theta_{n}, \lambda_{n}^{-\frac{1}{2}} A^{\frac{\beta}{2}}\left(A^{1-\alpha} u_{n}-\theta_{n}\right)\right\rangle+\lambda_{n}^{-1}\left\|A^{\frac{\beta}{2}} \theta_{n}\right\|^{2},
\end{aligned}
$$

where the penultimate equality is due to $(4.8 \mathrm{a})$.

It follows from (4.9) that

$$
\left\|\lambda_{n}^{-1} A^{\alpha-\frac{\gamma}{2}}\left(A^{1-\alpha} u_{n}-\theta_{n}\right)\right\|=O(1) .
$$

By taking the inner product of (4.20) with $A^{1-2 \alpha+\beta+\frac{\gamma}{2}} u_{n}$ in $H$, which is bounded due to $1-2 \alpha+\beta+\frac{\gamma}{2} \leq \frac{1}{2}$ in $R_{1}$, we arrive at

$$
\begin{aligned}
& \left\langle\lambda_{n}^{-1} A^{\alpha-\frac{\gamma}{2}}\left(A^{1-\alpha} u_{n}-\theta_{n}\right), A^{1-2 \alpha+\beta+\frac{\gamma}{2}} u_{n}\right\rangle \\
& \quad=\left\langle\lambda_{n}^{-\frac{1}{2}} A^{\alpha-\frac{\gamma}{2}}\left(A^{1-\alpha} u_{n}-\theta_{n}\right), \lambda_{n}^{-\frac{1}{2}}\left(A^{1-2 \alpha+\beta+\frac{\gamma}{2}} u_{n}-A^{-\alpha+\beta+\frac{\gamma}{2}} \theta_{n}\right\rangle\right. \\
& \quad+\left\langle\lambda_{n}^{-\frac{1}{2}} A^{\alpha-\frac{\gamma}{2}}\left(A^{1-\alpha} u_{n}-\theta_{n}\right), A^{-\alpha+\beta+\frac{\gamma}{2}} \theta_{n}\right\rangle \\
& \quad=\left\|\lambda_{n}^{-\frac{1}{2}} A^{\frac{\beta}{2}}\left(A^{1-\alpha} u_{n}-\theta_{n}\right)\right\|^{2}-\left\langle\lambda_{n}^{-\frac{1}{2}} A^{\frac{\beta}{2}}\left(A^{1-\alpha} u_{n}-\theta_{n}\right), \lambda_{n}^{-\frac{1}{2}} A^{\frac{\beta}{2}} \theta_{n}\right\rangle=O(1) .
\end{aligned}
$$




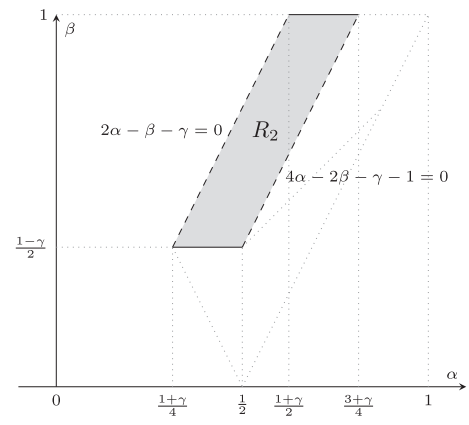

(a) $\gamma=\frac{1}{4}$

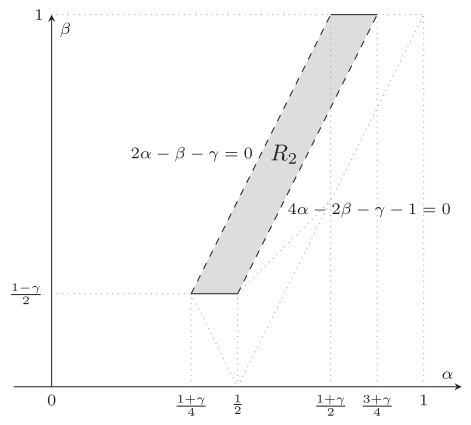

(b) $\gamma=\frac{1}{2}$

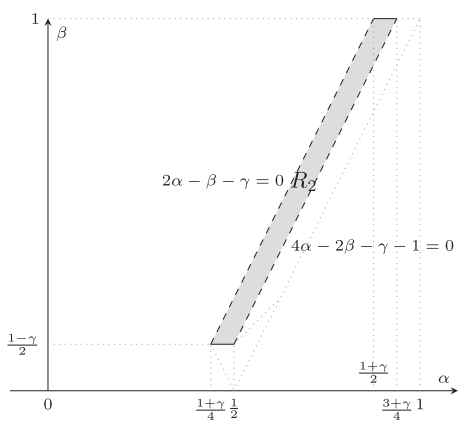

(c) $\gamma=\frac{3}{4}$

FiguRE 5. Visualization of $R_{2}$ at various $\gamma$ 's.

Therefore, by (4.10a) and (4.21), we have that

$$
\left\|\lambda_{n}^{-\frac{1}{2}} A^{\frac{\beta}{2}}\left(A^{1-\alpha} u_{n}-\theta_{n}\right)\right\|^{2}=O(1) .
$$

It follows from (4.10a), (4.19), and (4.22) that

$$
\left\|A^{\frac{1}{2}} u_{n}\right\|=o(1)
$$

which further implies in reference of (4.10a), (4.10b), and (4.15) that

$$
\left\|v_{n}\right\|^{2}+\left\|A^{\frac{\gamma}{2}} v_{n}\right\|^{2}=o(1) .
$$

From (4.17), (4.23), and (4.24), we have proved $\left\|U_{n}\right\|_{\mathcal{H}}=0$, which is a contradiction to (4.6).

Case 2. Let $(\alpha, \beta, \gamma) \in R_{2}$, where

$$
R_{2}=\left\{(\alpha, \beta, \gamma) \mid \frac{1-\gamma}{2} \leq \beta \leq 1,4 \alpha-2 \beta-\gamma-1<0,2 \alpha-\beta-\gamma>0,0 \leq \gamma<1\right\}
$$

and is shown in Figure 5 where $\gamma$ is $\frac{1}{4}, \frac{1}{2}$, and $\frac{3}{4}$, respectively.

Taking

$$
\mu=\frac{2(2 \alpha-\beta-\gamma)}{1-\gamma}
$$

Note that in $R_{2}, \frac{\gamma}{2}<\alpha-\frac{\beta}{2}<\frac{1}{2}$, where we have used $\gamma \in(0,1)$. Then, using the interpolation's inequality (4.1),

$$
\begin{aligned}
\left\|\lambda_{n}^{-\frac{\mu}{2}} A^{\alpha-\frac{\beta}{2}} v_{n}\right\| & \leq \lambda_{n}^{-\frac{\mu}{2}}\left\|A^{\frac{1}{2}} v_{n}\right\|^{\frac{\alpha-\frac{\beta}{2}-\frac{\gamma}{2}}{\frac{1}{2}-\frac{\gamma}{2}}}\left\|A^{\frac{\gamma}{2}} v_{n}\right\|^{\frac{\frac{1}{2}-\alpha+\frac{\beta}{2}}{\frac{1}{2}-\frac{\gamma}{2}}}=\lambda_{n}^{-\frac{\mu}{2}}\left\|A^{\frac{1}{2}} v_{n}\right\|^{\frac{2 \alpha-\beta-\gamma}{1-\gamma}}\left\|A^{\frac{\gamma}{2}} v_{n}\right\|^{\frac{1-2 \alpha+\beta}{1-\gamma}} \\
& =\lambda_{n}^{-\frac{\mu}{2}}\left\|A^{\frac{1}{2}} v_{n}\right\|^{\frac{2 \alpha-\beta-\gamma}{1-\gamma}}\left\|A^{\frac{\gamma}{2}} v_{n}\right\|^{1-\frac{2 \alpha-\beta-\gamma}{1-\gamma}}=\left\|\lambda_{n}^{-1} A^{\frac{1}{2}} v_{n}\right\|^{\frac{\mu}{2}}\left\|A^{\frac{\gamma}{2}} v_{n}\right\|^{1-\frac{\mu}{2}},
\end{aligned}
$$

where $\frac{\mu}{2} \in(0,1)$ in $S_{2}$, Hence, by (4.6) and (4.8a),

$$
\left\|\lambda_{n}^{-\frac{\mu}{2}} A^{\alpha-\frac{\beta}{2}} v_{n}\right\|=O(1)
$$


which, combined with (4.10a), further leads to

$$
\lambda_{n}^{-\mu}\left\langle A^{\frac{1}{2}} v_{n}, A^{\alpha-\frac{1}{2}} \theta_{n}\right\rangle=\left\langle\lambda_{n}^{-\frac{\mu}{2}} A^{\alpha-\frac{\beta}{2}} v_{n}, \lambda_{n}^{-\frac{\mu}{2}} A^{\frac{\beta}{2}} \theta_{n}\right\rangle=o(1) .
$$

It follows from (4.10d) and (4.27) that

$$
\left\|\lambda_{n}^{\frac{1-\mu}{2}} \theta_{n}\right\|=o(1) \Rightarrow\left\|\theta_{n}\right\|=o(1)
$$

Dividing (4.8c) by $\lambda_{n}^{\frac{1-\mu}{2}}$, and reference to (4.28), we have

$$
\lambda_{n}^{-\frac{1+\mu}{2}} A^{\alpha-\frac{1}{2}}\left(A^{\frac{1}{2}} v_{n}+A^{\beta-\alpha+\frac{1}{2}} \theta_{n}\right)=o(1) .
$$

On the other hand, when $\alpha \geq \frac{1}{2}$, take $b=\frac{1-\mu}{2}$ which is in $(0,1)$. It is clear that $\left\|\lambda_{n}^{-b} A^{1-\alpha} u_{n}\right\|=o(1)$ by (4.6). When $\alpha<\frac{1}{2}$, let $b=\frac{1-2 \alpha}{1-\gamma}$. Then $b \in(0,1)$ because in $R_{2}, 2 \alpha-\beta-\gamma>0 \Rightarrow 2 \alpha-\gamma>0 \Rightarrow 1-2 \alpha<$ $1-\gamma \Rightarrow b \in(0,1)$. Furthermore, when $\alpha<\frac{1}{2}$, we have that $\alpha-\frac{1}{2}<0<\alpha-\frac{\gamma}{2}$. Then, by interpolation we have

$$
\lambda_{n}^{-b}\left\|A^{1-\alpha} u_{n}-\theta_{n}\right\| \leq\left\|\lambda_{n}^{-1} A^{\alpha-\frac{\gamma}{2}}\left(A^{1-\alpha} u_{n}-\theta_{n}\right)\right\|^{b}\left\|A^{\alpha-\frac{1}{2}}\left(A^{1-\alpha} u_{n}-\theta_{n}\right)\right\|^{1-b}=O(1),
$$

since $\left\|\lambda_{n}^{-1} A^{\alpha-\frac{\gamma}{2}}\left(A^{1-\alpha} u_{n}-\theta_{n}\right)\right\|$ is bounded due to (4.9) and $\left\|A^{\alpha-\frac{1}{2}}\left(A^{1-\alpha} u_{n}-\theta_{n}\right)\right\|=\left\|A^{\frac{1}{2}} u_{n}-A^{\alpha-\frac{1}{2}} \theta_{n}\right\| \leq$ $\left\|A^{\frac{1}{2}} u_{n}\right\|+\left\|A^{\alpha-\frac{1}{2}} \theta_{n}\right\|$ is also bounded because we are considering $\alpha<\frac{1}{2}$. In this case we also obtain the boundedness of $\left\|\lambda_{n}^{-b} A^{1-\alpha} u_{n}\right\|$ because $\left\|\lambda_{n}^{-b} \theta_{n}\right\|$ is bounded.

For both $\alpha \geq \frac{1}{2}$ and $\alpha<\frac{1}{2}$, the inner product of (4.29) with $\lambda_{n}^{-b} A^{1-\alpha} u_{n}$ gives

$$
\lambda_{n}^{-\frac{1+\mu}{2}-b}\left\langle A^{\frac{1}{2}} v_{n}, A^{\frac{1}{2}} u_{n}\right\rangle+\lambda_{n}^{-\frac{1+\mu}{2}-b}\left\langle A^{\frac{\beta}{2}} \theta_{n}, A^{1-\alpha+\frac{\beta}{2}} u_{n}\right\rangle=o(1) .
$$

We can multiple (4.31) by $\lambda_{n}^{-1+\frac{1+\mu}{2}+b}$ to get

$$
\begin{gathered}
\left\langle\lambda_{n}^{-1} A^{\frac{1}{2}} v_{n}, A^{\frac{1}{2}} u_{n}\right\rangle+\lambda_{n}^{-1}\left\langle A^{\frac{\beta}{2}} \theta_{n}, A^{1-\alpha+\frac{\beta}{2}} u_{n}\right\rangle=o(1) \\
\Rightarrow \quad i\left\|A^{\frac{1}{2}} u_{n}\right\|^{2}+\left\langle\lambda_{n}^{-\frac{\mu}{2}} A^{\frac{\beta}{2}} \theta_{n}, \lambda_{n}^{-1+\frac{\mu}{2}} A^{1-\alpha+\frac{\beta}{2}} u_{n}\right\rangle=o(1),
\end{gathered}
$$

where we have used the fact that for $\alpha \geq \frac{1}{2}, \frac{1+\mu}{2}+b=1$; and for $\alpha<\frac{1}{2}, \frac{1+\mu}{2}+b=\frac{3}{2}-\frac{\beta}{1-\gamma} \leq 1$ because $\beta \geq \frac{1-\gamma}{2}$ in $R_{2}$.

On the other hand,

$$
\begin{aligned}
& \left\|\lambda_{n}^{-1+\frac{\mu}{2}} A^{1-\alpha+\frac{\beta}{2}} u_{n}\right\|=\left\|\lambda_{n}^{-1+\frac{\mu}{2}} A^{\frac{\beta}{2}}\left(A^{1-\alpha} u_{n}-\theta_{n}\right)+\lambda_{n}^{-1+\frac{\mu}{2}} A^{\frac{\beta}{2}} \theta_{n}\right\| \\
\leq & \left\|\lambda_{n}^{-1+\frac{\mu}{2}} A^{\frac{\beta}{2}}\left(A^{1-\alpha} u_{n}-\theta_{n}\right)\right\|+\left\|\lambda_{n}^{-1+\frac{\mu}{2}} A^{\frac{\beta}{2}} \theta_{n}\right\|=\left\|\lambda_{n}^{-1+\frac{\mu}{2}} A^{\frac{\beta}{2}}\left(A^{1-\alpha} u_{n}-\theta_{n}\right)\right\|+o(1),
\end{aligned}
$$

where for the last equality we have used (4.10a) and the fact that $-1+\frac{\mu}{2}<-\frac{\mu}{2}$ because $\mu<1$ by the fact that $4 \alpha-2 \beta-\gamma-1<0$ in $R_{2}$. Furthermore, let $c=\frac{1-2 \alpha+\beta}{1-\gamma}$, by $\alpha-\frac{1}{2}<\frac{\beta}{2}<\alpha-\frac{\gamma}{2}$ in $R_{2}$ and (4.30),

$$
\begin{aligned}
& \lambda_{n}^{-1+\frac{\mu}{2}}\left\|A^{\frac{\beta}{2}}\left(A^{1-\alpha} u_{n}-\theta_{n}\right)\right\| \\
\leq & \lambda_{n}^{-1+\frac{\mu}{2}+c}\left\|\lambda_{n}^{-1} A^{\alpha-\frac{\gamma}{2}}\left(A^{1-\alpha} u_{n}-\theta_{n}\right)\right\|^{c}\left\|A^{\alpha-\frac{1}{2}}\left(A^{1-\alpha} u_{n}-\theta_{n}\right)\right\|^{1-c}=O(1),
\end{aligned}
$$

where $-1+\frac{\mu}{2}+c=0$ and hence $c \in(0,1)$. 


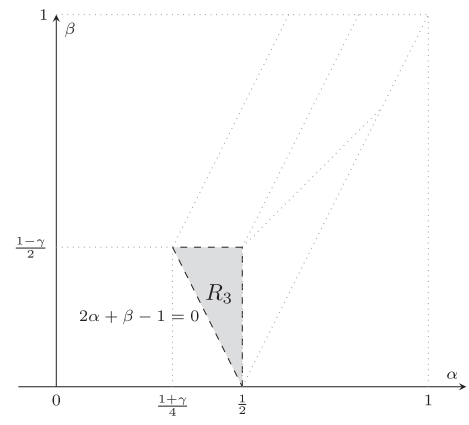

(a) $\gamma=\frac{1}{4}$

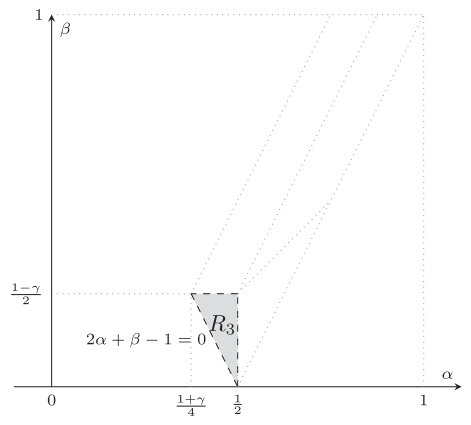

(b) $\gamma=\frac{1}{2}$

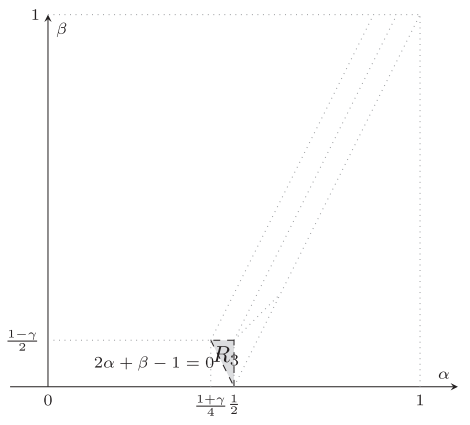

(c) $\gamma=\frac{3}{4}$

Figure 6. Visualization of $R_{3}$ at various $\gamma$ 's.

Applying (4.10a), (4.33), and (4.34) to (4.32), we conclude that

$$
\left\|A^{\frac{1}{2}} u_{n}\right\|=o(1)
$$

With(4.10a), (4.10b), (4.26), (4.28), and (4.35), and by an argument similar to that presented in Case 1, we have proved $\left\|U_{n}\right\|_{\mathcal{H}}=0$, which is a contradiction to (4.6).

Case 3. Let $(\alpha, \beta, \gamma) \in R_{3}$, where

$$
R_{3}=\left\{(\alpha, \beta, \gamma) \mid \alpha<\frac{1}{2}, \beta<\frac{1-\gamma}{2}, 2 \alpha+\beta-1>0,0 \leq \gamma<1\right\}
$$

and is shown in Figure 6 where $\gamma$ is $\frac{1}{4}, \frac{1}{2}$, and $\frac{3}{4}$, respectively.

Take $\mu=\frac{2(2 \alpha+\beta-1)}{1-\gamma}$. Then $\mu \in(0,1) . \mu>0$ is due to $2 \alpha+\beta-1>0$ and $\gamma<1$ in $R_{3}$. To see $\mu<1$, consider $\beta<\frac{1-\gamma}{2} \Rightarrow \frac{1}{\beta}>\frac{2}{1-\gamma} \Rightarrow 1>\frac{2 \alpha+\beta-1}{\beta}>\frac{2(2 \alpha+\beta-1)}{1-\gamma}=\mu$, where $1>\frac{2 \alpha+\beta-1}{\beta}$ is due to $\alpha<\frac{1}{2}$ in $R_{3}$.

Note that $\frac{\gamma}{2}<\alpha-\frac{\beta}{2}<\frac{1}{2}$ in $R_{3}$, where the first inequality holds because $\alpha>\frac{1-\beta}{2}>\frac{\beta+\gamma}{2}$ due to the fact that $2 \alpha+\beta-1>0$ and $\beta<\frac{1-\gamma}{2}$ in $R_{3}$, and the second inequality is due to $\alpha<\frac{1}{2}$ in $R_{3}$. Let $a=\frac{2 \alpha-\beta-\gamma}{1-\gamma}$. Then $a \in(0,1)$ because the foregoing inequality $\frac{\gamma}{2}<\alpha-\frac{\beta}{2}<\frac{1}{2}$.

$$
\lambda_{n}^{-a}\left\|A^{\alpha-\frac{\beta}{2}} v_{n}\right\| \leq\left\|\lambda_{n}^{-1} A^{\frac{1}{2}} v_{n}\right\|^{a}\left\|A^{\frac{\gamma}{2}} v_{n}\right\|^{1-a}=O(1) .
$$

Note that $a-\frac{\mu}{2}=1-\frac{2 \beta}{1-\gamma}>0$ because $\beta<\frac{1-\gamma}{2}$, and $1-\frac{\mu}{2}-a=\frac{2-4 \alpha}{1-\gamma}>0$ because $\alpha<\frac{1}{2}$ and $\gamma<1$. Since $a-\frac{\mu}{2}>0$, dividing (4.10d) by $\lambda_{n}^{a-\frac{\mu}{2}}$ yields,

$$
\begin{aligned}
o(1) & =\lambda_{n}^{1-\frac{\mu}{2}-a}\left\|\theta_{n}\right\|^{2}+\lambda_{n}^{-\frac{\mu}{2}-a}\left\langle A^{\frac{1}{2}} v_{n}, A^{\alpha-\frac{1}{2}} \theta_{n}\right\rangle=\lambda_{n}^{1-\frac{\mu}{2}-a}\left\|\theta_{n}\right\|^{2}+\left\langle\lambda_{n}^{-a} A^{\alpha-\frac{\beta}{2}} v_{n}, \lambda_{n}^{-\frac{\mu}{2}} A^{\frac{\beta}{2}} \theta_{n}\right\rangle \\
& =\lambda_{n}^{1-\frac{\mu}{2}-a}\left\|\theta_{n}\right\|^{2}+o(1) \Rightarrow\left\|\lambda_{n}^{\frac{1}{2}-\frac{\mu}{4}-\frac{a}{2}} \theta_{n}\right\|^{2}=o(1) \Rightarrow\left\|\theta_{n}\right\|^{2}=o(1),
\end{aligned}
$$

where we have used (4.10a) and (4.36) to show $\left\langle\lambda_{n}^{-a} A^{\alpha-\frac{\beta}{2}} v_{n}, \lambda_{n}^{-\frac{\mu}{2}} A^{\frac{\beta}{2}} \theta_{n}\right\rangle=o(1)$. 


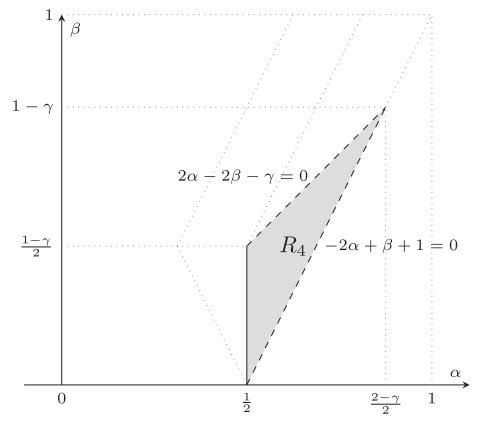

(a) $\gamma=\frac{1}{4}$

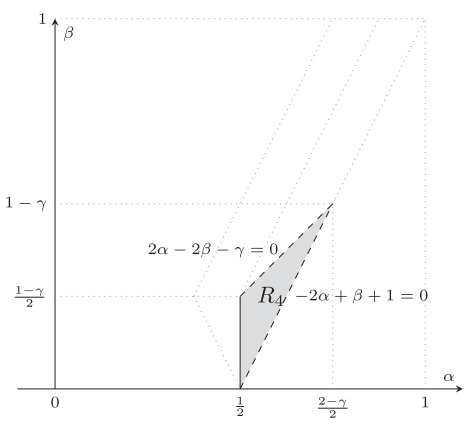

(b) $\gamma=\frac{1}{2}$

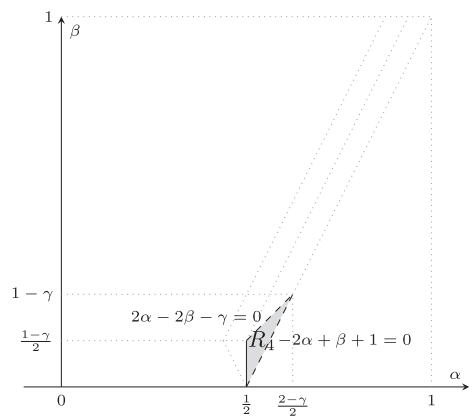

(c) $\gamma=\frac{3}{4}$

FiguRe 7. Visualization of $R_{4}$ at various $\gamma$ 's.

Note that $\frac{1}{2}-\frac{3 \mu}{4}+\frac{a}{2}=\frac{2-2 \alpha-2 \beta-\gamma}{1-\gamma}>0$ because $\gamma<1$ and $1-\gamma-2 \beta>0>2 \alpha-1$ in $R_{3}$. Dividing (4.8c) by $\lambda_{n}^{\frac{1}{2}-\frac{3 \mu}{4}+\frac{a}{2}}$ and in reference of (4.37), we obtain

$$
\begin{aligned}
& i \lambda_{n}^{\frac{1}{2}-\frac{\mu}{4}-\frac{a}{2}} \theta_{n}+\lambda_{n}^{-\frac{1}{2}-\frac{\mu}{4}-\frac{a}{2}} A^{\alpha-\frac{1}{2}}\left(A^{\frac{1}{2}} v_{n}+A^{\beta-\alpha+\frac{1}{2}} \theta_{n}\right)=o(1) \\
\Rightarrow & \lambda_{n}^{-\frac{1}{2}-\frac{\mu}{4}-\frac{a}{2}}\left(A^{\alpha} v_{n}+A^{\beta} \theta_{n}\right)=o(1) .
\end{aligned}
$$

Note that $-\frac{1}{2}+\frac{\mu}{4}+\frac{a}{2}=\frac{2 \alpha-1}{1-\gamma}=-b$ in (4.30). Because $\alpha-\frac{1}{2}<0<\alpha-\frac{\gamma}{2}$ still holds, where we have used $\frac{\gamma}{2}<\alpha-\frac{\beta}{2}$ for to show $0<\alpha-\frac{\gamma}{2}$, (4.30) is still true for $R_{3}$. Then, $\left\|\lambda_{n}^{-\frac{1}{2}+\frac{\mu}{4}+\frac{a}{2}} A^{1-\alpha} u_{n}\right\|=O(1)$ by $(4.30)$. Taking the inner product of (4.38) with $\lambda_{n}^{-\frac{1}{2}+\frac{\mu}{4}+\frac{a}{2}} A^{1-\alpha} u_{n}$ in $H$ yields,

$$
\begin{aligned}
& \left\langle A^{\frac{1}{2}} u_{n}, \lambda_{n}^{-1} A^{\frac{1}{2}} v_{n}\right\rangle+\left\langle\lambda_{n}^{-1+\frac{\mu}{2}} A^{1-\alpha+\frac{\beta}{2}} u_{n}, \lambda_{n}^{-\frac{\mu}{2}} A^{\frac{\beta}{2}} \theta_{n}\right\rangle=o(1) \\
\Rightarrow & i\left\|A^{\frac{1}{2}} u_{n}\right\|^{2}+\left\langle\lambda_{n}^{-1+\frac{\mu}{2}} A^{1-\alpha+\frac{\beta}{2}} u_{n}, \lambda_{n}^{-\frac{\mu}{2}} A^{\frac{\beta}{2}} \theta_{n}\right\rangle=o(1),
\end{aligned}
$$

where we have used (4.10a) and $\mu \in(0,1)$.

Again, we only need to show the boundedness of $\left\|\lambda_{n}^{-1+\frac{\mu}{2}} A^{1-\alpha+\frac{\beta}{2}} u_{n}\right\|$ to get $\left\|A^{\frac{1}{2}} u_{n}\right\|=o(1)$. By (4.34), $\lambda_{n}^{-1+\frac{\mu}{2}}\left(A^{1-\alpha+\frac{\beta}{2}} u_{n}-A^{\frac{\beta}{2}} \theta_{n}\right)=o(1)$ because $-1+\frac{\mu}{2}+c=-1+\frac{2 \beta}{1-\gamma}<0$. Therefore, $\lambda_{n}^{-1+\frac{\mu}{2}} A^{1-\alpha+\frac{\beta}{2}} u_{n}=$ $\lambda_{n}^{-1+\frac{\mu}{2}} A^{\frac{\beta}{2}} \theta_{n}=o(1)$, where we have used the fact that $-1+\frac{\mu}{2}<-\frac{\mu}{2}$ and (4.10a).

With(4.10a), (4.10b), (4.36), (4.37), and (4.39), and by an argument similar to that presented in Case 1, we have proved $\left\|U_{n}\right\|_{\mathcal{H}}=0$, which is a contradiction to (4.6).

Case 4. Let $(\alpha, \beta, \gamma) \in R_{4}$, where

$$
R_{4}=\left\{(\alpha, \beta, \gamma) \mid \alpha \geq \frac{1}{2}, 2 \alpha-2 \beta-\gamma>0,-2 \alpha+\beta+1>0,0 \leq \gamma<1,\right\}
$$

and is shown in Figure 7 where $\gamma$ is $\frac{1}{4}, \frac{1}{2}$, and $\frac{3}{4}$, respectively.

Take $\mu=\frac{2 \beta}{2 \alpha-\gamma}$, then $\mu \in(0,1)$ because $2 \alpha-2 \beta-\gamma>0$ in $R_{4}$. Note that $\beta-\alpha+\frac{\gamma}{2}<0$ in $R_{4}$. Thus,

$$
\left\|A^{\frac{\gamma}{2}} v_{n}+A^{\beta-\alpha+\frac{\gamma}{2}} \theta_{n}\right\|=O(1) .
$$


On the other hand,

$$
\left\|\lambda_{n}^{-1} A^{\alpha-\frac{\gamma}{2}}\left(A^{\frac{\gamma}{2}} v_{n}+A^{\beta-\alpha+\frac{\gamma}{2}}\right) \theta_{n}\right\|=O(1)
$$

by (4.8c). Acting $A^{\beta-\alpha+\frac{\gamma}{2}}$ to (4.8c) yields,

$$
\lambda_{n}^{1-\mu} A^{\beta-\alpha+\frac{\gamma}{2}} \theta_{n}+\lambda_{n}^{-\mu} A^{\beta}\left(A^{\frac{\gamma}{2}} v_{n}+A^{\beta-\alpha+\frac{\gamma}{2}} \theta_{n}\right)=o(1)
$$

Since $\alpha-\frac{\gamma}{2}>\beta \geq 0$ in $R_{4}$, by interpolation, we obtain,

$$
\lambda_{n}^{-\mu}\left\|A^{\beta}\left(A^{\frac{\gamma}{2}} v_{n}+A^{\beta-\alpha+\frac{\gamma}{2}} \theta_{n}\right)\right\| \leq\left\|\lambda_{n}^{-1} A^{\alpha-\frac{\gamma}{2}}\left(A^{\frac{\gamma}{2}} v_{n}+A^{\beta-\alpha+\frac{\gamma}{2}} \theta_{n}\right)\right\|^{\mu}\left\|A^{\frac{\gamma}{2}} v_{n}+A^{\beta-\alpha+\frac{\gamma}{2}} \theta_{n}\right\|^{1-\mu} .
$$

By (4.40), (4.41), and (4.43),

$$
\lambda_{n}^{-\mu}\left\|A^{\beta}\left(A^{\frac{\gamma}{2}} v_{n}+A^{\beta-\alpha+\frac{\gamma}{2}} \theta_{n}\right)\right\|=O(1) .
$$

It follows from (4.42) and (4.44) that

$$
\left\|\lambda_{n}^{1-\mu} A^{\beta-\alpha+\frac{\gamma}{2}} \theta_{n}\right\|=O(1)
$$

Let $b=\frac{2 \alpha-2 \beta-\gamma}{2 \alpha-\beta-\gamma} \in(0,1]$ in $R_{4}$. It is straightforward to verify the following equality

$$
-\frac{\mu}{2} b+(1-\mu)(1-b)=0
$$

Then, with $0<\alpha-\beta-\frac{\gamma}{2}<\alpha-\frac{\beta}{2}-\frac{\gamma}{2}$ and by interpolation,

$$
\begin{aligned}
\left\|\theta_{n}\right\| & =\left\|A^{\alpha-\beta-\frac{\gamma}{2}}\left(A^{\beta-\alpha+\frac{\gamma}{2}} \theta_{n}\right)\right\| \leq\left\|A^{\alpha-\frac{\beta}{2}-\frac{\gamma}{2}}\left(A^{\beta-\alpha+\frac{\gamma}{2}} \theta_{n}\right)\right\|^{b}\left\|A^{\beta-\alpha+\frac{\gamma}{2}} \theta_{n}\right\|^{1-b} \\
& =\left\|A^{\frac{\beta}{2}} \theta_{n}\right\|^{b}\left\|A^{\beta-\alpha+\frac{\gamma}{2}} \theta_{n}\right\|^{1-b}=\left\|\lambda_{n}^{-\frac{\mu}{2}} A^{\frac{\beta}{2}} \theta_{n}\right\|^{b}\left\|\lambda_{n}^{1-\mu} A^{\beta-\alpha+\frac{\gamma}{2}} \theta_{n}\right\|^{1-b}=o(1),
\end{aligned}
$$

where the penultimate equality is due to (4.46).

Next, note that $1-\alpha<\frac{1}{2}$ in $R_{4}$, which implies that $\left\|A^{1-\alpha} u_{n}\right\|=O(1)$. Here, we have used (4.8a). Taking inner product of (4.8c) with $\lambda_{n}^{\mu-1} A^{1-\alpha} u_{n}$ yields,

$$
\begin{aligned}
o(1) & =i\left\langle A^{1-\alpha} u_{n}, \theta_{n}\right\rangle+\left\langle\lambda_{n}^{-1} A^{\frac{1}{2}}\left(A^{\frac{1}{2}} v_{n}+A^{\beta-\alpha+\frac{1}{2}} \theta_{n}\right), u_{n}\right\rangle \\
& =\left\langle\lambda_{n}^{-1} A^{\frac{1}{2}} v_{n}, A^{\frac{1}{2}} u_{n}\right\rangle+\left\langle\lambda_{n}^{-1} A^{\beta-\alpha+\frac{1}{2}} \theta_{n}, A^{\frac{1}{2}} u_{n}\right\rangle=i\left\|A^{\frac{1}{2}} u_{n}\right\|^{2}+o(1),
\end{aligned}
$$

where we have used (4.6), (4.8a), (4.47), $\beta-\alpha+\frac{1}{2}<\frac{\beta}{2}$, and $-1<-\frac{\mu}{2}$ to obtain the last equality.

Finally, we take inner product of (4.8c) with $\lambda_{n}^{\mu-1} \theta_{n}$ to get

$$
o(1)=i\left\|\theta_{n}\right\|^{2}+\lambda_{n}^{-1}\left\langle A^{\frac{1}{2}} v_{n}, A^{\alpha-\frac{1}{2}} \theta_{n}\right\rangle+\lambda_{n}^{-1}\left\|A^{\frac{\beta}{2}} \theta_{n}\right\|^{2}=\left\langle\lambda_{n}^{-1} A^{\frac{1}{2}} v_{n}, A^{\alpha-\frac{1}{2}} \theta_{n}\right\rangle,
$$

where the last equality is obtained similarly to that in (4.48).

With (4.10b), (4.47), (4.48), and (4.49), and by an argument similar to that presented in Case 1, we have proved $\left\|U_{n}\right\|_{\mathcal{H}}=0$, which is a contradiction to (4.6). 


\section{Asymptotic Behavior of eigenvalues}

In this section, we are going to study the asymptotic behavior of some eigenvalue sequences for the operator $\mathcal{A}_{\alpha, \beta, \gamma}$. Recall that we assume there exists a sequence $\mu_{n}$ of eigenvalues of $A$ such that

$$
0<\mu_{1} \leq \mu_{2} \leq \cdots, \quad \lim _{n \rightarrow \infty} \mu_{n}=\infty
$$

The following lemma is a direct consequence.

Lemma 5.1. Let

$$
f(\lambda, \mu)=\left(1+\kappa \mu^{\gamma}\right) \lambda^{3}+\left(\kappa \mu^{\gamma+\beta}+\mu^{\beta}\right) \lambda^{2}+\left(\mu+\mu^{2 \alpha}\right) \lambda+\mu^{\beta+1}, \quad \forall(\lambda, \mu) \in \mathbb{C} \times \mathbb{R}_{+},
$$

with $\kappa=1$. If the following holds:

$$
f\left(\lambda_{n}, \mu_{n}\right)=0
$$

then $\lambda_{n}$ is an eigenvalue of $\mathcal{A}_{\alpha, \beta, \gamma}$.

Now, for any $n \geq 1$, we consider the following equation:

$$
f\left(\lambda, \mu_{n}\right) \equiv\left(1+\mu_{n}^{\gamma}\right) \lambda^{3}+\left(\mu_{n}^{\gamma+\beta}+\mu_{n}^{\beta}\right) \lambda^{2}+\left(\mu_{n}^{2 \alpha}+\mu_{n}\right) \lambda+\mu_{n}^{\beta+1}=0 .
$$

Let us denote

$$
a_{n}=1+\mu_{n}^{\gamma}, \quad b_{n}=\mu_{n}^{\gamma+\beta}+\mu^{\beta}, \quad c_{n}=\mu_{n}^{2 \alpha}+\mu_{n}, \quad d_{n}=\mu_{n}^{\beta+1} .
$$

Then (5.3) takes the following form:

$$
a_{n} \lambda^{3}+b_{n} \lambda^{2}+c_{n} \lambda+d_{n}=0
$$

with $a_{n}, b_{n}, c_{n}, d_{n} \in \mathbb{R}_{+}$. Let

$$
h_{n}=3 a_{n} c_{n}-b_{n}^{2}, \quad g_{n}=2 b_{n}^{3}-9 a_{n} b_{n} c_{n}+27 a_{n}^{2} d_{n}, \quad p_{n}=\frac{h_{n}}{3 a_{n}^{2}}, \quad q_{n}=\frac{g_{n}}{27 a_{n}^{3}} .
$$

Define

$$
\Delta_{n}=\left(\frac{q_{n}}{2}\right)^{2}+\left(\frac{p_{n}}{3}\right)^{3}=\frac{W_{n}}{108 a_{n}^{4}}, \text { where } W_{n}=27 a_{n}^{2} d_{n}^{2}-18 a_{n} b_{n} c_{n} d_{n}+4 a_{n} c_{n}^{3}+4 b_{n}^{3} d_{n}-b_{n}^{2} c_{n}^{2} .
$$

We also define

$$
\Phi_{n, \pm}=-\frac{q_{n}}{2} \pm \sqrt{\Delta_{n}} \equiv-\frac{q_{n}}{2} \pm \sqrt{\left(\frac{q_{n}}{2}\right)^{2}+\left(\frac{p_{n}}{3}\right)^{3}} .
$$

In our paper, for any $\xi=|\xi| e^{i \theta}$, we define $\xi^{\frac{1}{2}}=|\xi|^{\frac{1}{2}} e^{i \frac{\theta}{2}}$, and $\xi^{\frac{1}{3}}=|\xi|^{\frac{1}{3}} e^{i \frac{\theta}{3}}$. Note that since $a_{n}, b_{n}, c_{n}, d_{n} \in \mathbb{R}_{+}$, $\sqrt{\Delta_{n}}$ is either real or purely imaginary. Also notice that $\Phi_{n,+} \Phi_{n,-}=-\left(\frac{p_{n}}{3}\right)^{3}$. Since $p_{n}$ is real, we have that 
$\Phi_{n,+}^{\frac{1}{3}} \Phi_{n,-}^{\frac{1}{3}}=-\frac{p_{n}}{3}$. This statement is obviously true when $\sqrt{\Delta_{n}}$ is real. On the other hand, when $\sqrt{\Delta_{n}}$ is imaginary, $\Phi_{n,+}^{\frac{1}{3}}$ and $\Phi_{n,-}^{\frac{1}{3}}$ are complex conjugate so the statement still holds. Notice that

$$
\Phi_{n,+}+\Phi_{n,-}=-q_{n}, \quad \Phi_{n,+}-\Phi_{n,-}=2 \sqrt{\Delta_{n}}
$$

We can define $A_{n}$ and $B_{n}$ as:

$$
A_{n}=\Phi_{n,+}^{\frac{1}{3}}+\Phi_{n,-}^{\frac{1}{3}}=\frac{-q_{n}}{\Phi_{n,+}^{\frac{2}{3}}-\Phi_{n,+}^{\frac{1}{3}} \Phi_{n,-}^{\frac{1}{3}}+\Phi_{n,-}^{\frac{2}{3}}}, B_{n}=\Phi_{n,+}^{\frac{1}{3}}-\Phi_{n,-}^{\frac{1}{3}}=\frac{2 \sqrt{\Delta_{n}}}{\Phi_{n,+}^{\frac{2}{3}}+\Phi_{n,+}^{\frac{1}{3}} \Phi_{n,-}^{\frac{1}{3}}+\Phi_{n,-}^{\frac{2}{3}}}
$$

With the above notations, we have the following result ([15]) regarding the roots of (5.5).

Lemma 5.2 (Cardano's formula). Equation (5.5) admits three roots which are given by the following:

$$
\left\{\begin{array}{l}
\lambda_{n, 0}=A_{n}-\frac{b_{n}}{3 a_{n}} \\
\lambda_{n, \pm}=-\frac{1}{2} A_{n}-\frac{b_{n}}{3 a_{n}} \pm i \frac{\sqrt{3}}{2} B_{n}
\end{array}\right.
$$

As mentioned in the beginning of this section, we are interested in the asymptotic behavior of some eigenvalue sequences for the operator $\mathcal{A}_{\alpha, \beta, \gamma}$. Specifically, with the Cardano's formula, we are interested in characterizing the leading terms of $\lambda_{n, 0}$ and $\lambda_{n, \pm}$ as $n \rightarrow \infty$. This will be the focus of the rest of the section.

For the ease of presentation, we first partition

$$
G=\{(\alpha, \beta, \gamma) \mid 0 \leq \alpha \leq 1,0 \leq \beta \leq 1,0<\gamma \leq 1\}
$$

into various regions, and report the leading terms of $\lambda_{n, 0}$ and $\lambda_{n, \pm}$ in corresponding regions in Section 5.1. It should be noticed that $E \subseteq G$. Therefore, by identifying the leading terms of $\lambda_{n, 0}$ and $\lambda_{n, \pm}$ as in $G$, we will have complete knowledge of these leading terms in $E$. The leading terms of $\lambda_{n, 0}$ and $\lambda_{n, \pm}$ in $E$ are also reported in Section 5.1.

As of how we can determine the leading terms of $\lambda_{n, 0}$ and $\lambda_{n, \pm}$, based on the Cardano's Formula (5.10), we start from determining the leading terms of $A_{n}$ and $B_{n}$ as $n \rightarrow \infty$. This is the focus in Section 5.2.

In our derivation, we will show that just using the leading term of $A_{n}$ does not suffice to estimate the leading terms of $\lambda_{n, 0}$ and $\lambda_{n, \pm}$ as desired. This motivates us to further characterize the lower order terms of $A_{n}$, which will be the focus of Section 5.3. Upon acquiring these lower order terms, we can compute the leading terms of $\lambda_{n, 0}$ and $\lambda_{n, \pm}$, which is reported in Section 5.4.

\subsection{Partition of $G$ and leading terms of $\lambda_{n, 0}$ and $\lambda_{n, \pm}$}

The partition of $G$ is given by Definition 5.3. 


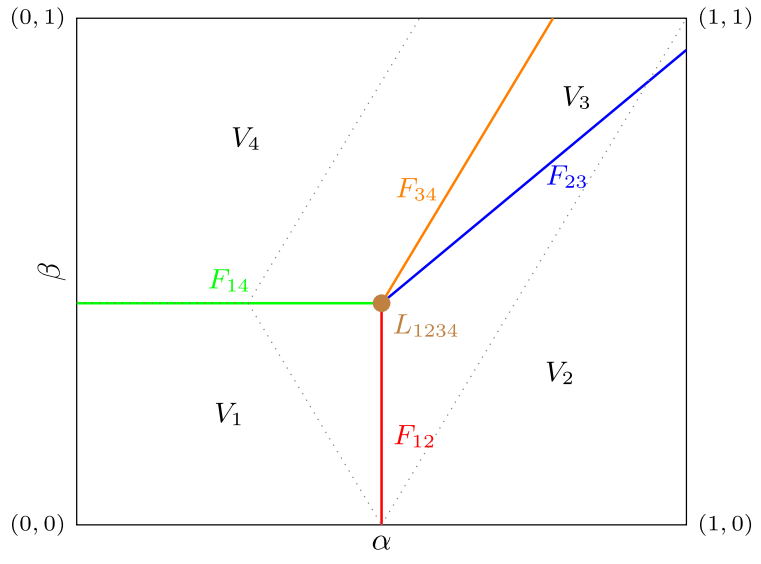

(a) IDs and locations of various regions.

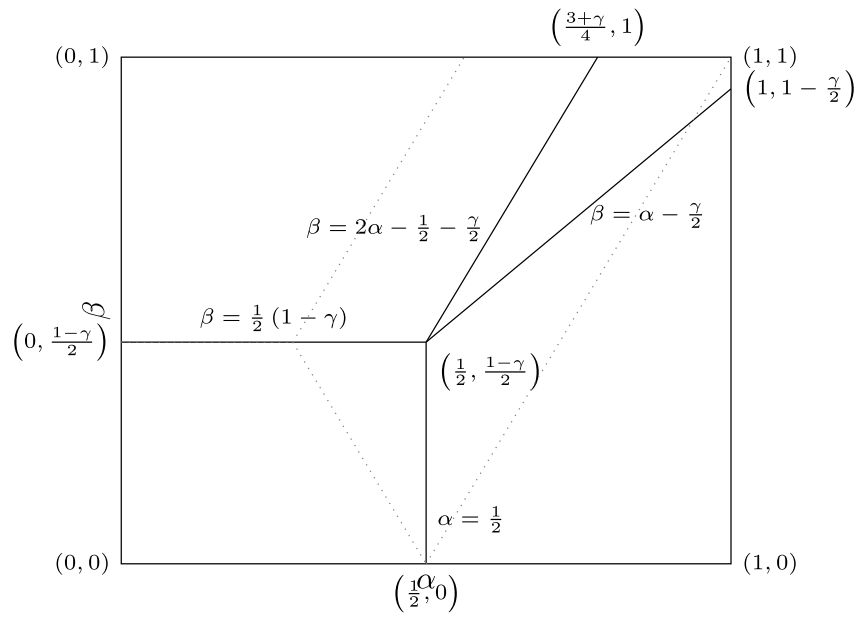

(b) boundaries of various regions.

Figure 8. Partition of $G$ according to Definition 5.3, choosing $\gamma=\frac{1}{8}$. The dotted lines represent the contours of various regions in $E$.

Definition 5.3. $G=\{(\alpha, \beta, \gamma) \mid 0 \leq \alpha \leq 1,0 \leq \beta \leq 1,0<\gamma \leq 1\}$ is partitioned into various regions given in (5.12). The parameter space $E$ considered in this paper is a subset of $G$.

$$
\left\{\begin{array}{l}
V_{1}=\left\{(\alpha, \beta, \gamma) \mid 0 \leq \alpha<\frac{1}{2}, 0 \leq \beta<\frac{1}{2}(1-\gamma), 0<\gamma<1\right\} \\
V_{2}=\left\{(\alpha, \beta, \gamma) \mid \frac{1}{2}<\alpha \leq 1,0 \leq \beta<\frac{1}{2}(2 \alpha-\gamma), 0<\gamma \leq 1\right\} \\
V_{3}=\left\{(\alpha, \beta, \gamma) \mid \frac{1}{4}(1+2 \beta+\gamma)<\alpha<1, \frac{1}{2}(2 \alpha-\gamma)<\beta \leq 1,0<\gamma<1\right\} \\
F_{3}=\left\{(\alpha, \beta, \gamma) \mid \frac{1}{4}(2+2 \beta)<\alpha<1, \frac{1}{2}(2 \alpha-1)<\beta<1, \gamma=1\right\} \\
V_{4}=\left\{(\alpha, \beta, \gamma) \mid 0 \leq \alpha<\frac{1}{4}(1+2 \beta+\gamma), \frac{1}{2}(1-\gamma)<\beta \leq 1,0<\gamma \leq 1\right\} \\
F_{12}=\left\{(\alpha, \beta, \gamma) \mid \alpha=\frac{1}{2}, 0 \leq \beta<\frac{1}{2}(1-\gamma), 0<\gamma<1\right\} \\
F_{14}=\left\{(\alpha, \beta, \gamma) \mid 0 \leq \alpha<\frac{1}{2}, \beta=\frac{1}{2}(1-\gamma), 0<\gamma \leq 1\right\} \\
F_{23}=\left\{(\alpha, \beta, \gamma) \mid \frac{1}{2}<\alpha \leq 1, \beta=\frac{1}{2}(2 \alpha-\gamma), 0<\gamma \leq 1\right\} \\
F_{34}=\left\{(\alpha, \beta, \gamma) \mid \alpha=\frac{1}{4}(1+2 \beta+\gamma), \frac{1}{2}(1-\gamma)<\beta \leq 1,0<\gamma \leq 1\right\} \\
L_{1234}=\left\{(\alpha, \beta, \gamma) \mid \alpha=\frac{1}{2}, \beta=\frac{1}{2}(1-\gamma), 0<\gamma \leq 1\right\}
\end{array}\right.
$$

Note that all the regions in (5.12) forms a partition of $G$. The notation used in Definition 5.3 is suggestive. We use $V_{i}$ to represent a volume (polytope), where $i \in\{1,2,3,4\}$. We use $S$ and the corresponding subscripts to represent a surface (plane) that is the common boundary between two volumes. For example, $F_{12}$ represents the surface that is the common boundary between $V_{1}$ and $V_{2}$. Finally we use $L$ and the corresponding subscripts to represent a line segment generated by the intersection of multiple volumes. Figure 8 visualizes the IDs, location, and the geometries of various regions by partitioning $G$. Figure 9 shows how the partition changes at various $\gamma$ levels.

The leading terms of $\lambda_{n, 0}$ and $\lambda_{n, \pm}$ in these various regions of $G$ are given by Theorem 5.4.

Theorem 5.4. $\lambda_{n, 0}$ and $\lambda_{n, \pm}$ are given respectively in the regions of $G$ defined in Definition 5.3 as: 


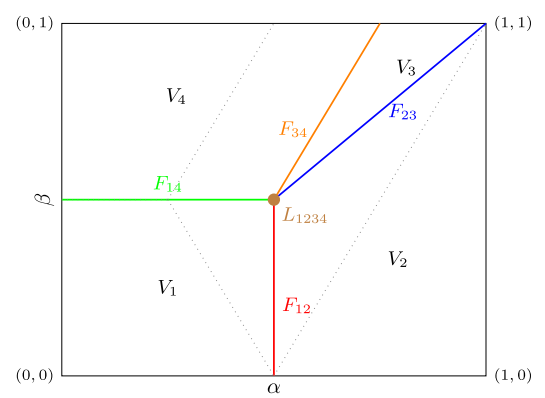

(a) $\gamma=0$

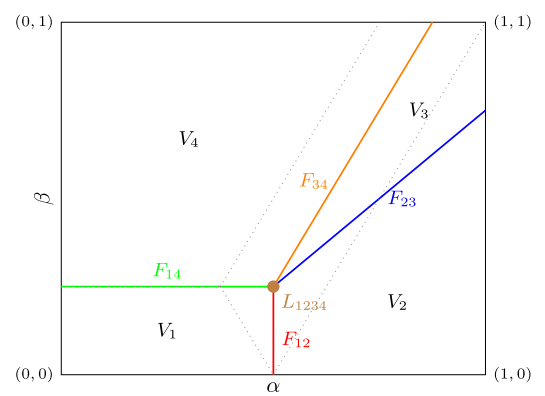

(b) $\gamma=\frac{1}{2}$

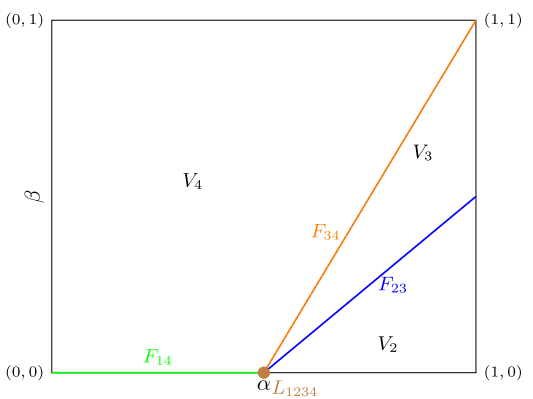

(c) $\gamma=1$

Figure 9. Partitions of $G$ at various $\gamma$ 's. The dotted lines represent the contours of various regions in $E$. When $\gamma=1$, the contours overlapped with $F_{14}$ and $F_{34}$.

- When $(\alpha, \beta, \gamma) \notin L_{1234}$,

$$
\begin{cases}\lambda_{n, 0}=-\mu_{n}^{\beta}(1+o(1)), \quad \lambda_{n, \pm}=-\frac{1}{2} \mu_{n}^{2 \alpha+\beta-1}(1+o(1)) \pm i \mu_{n}^{\frac{1}{2}-\frac{\gamma}{2}}(1+o(1)), & (\alpha, \beta, \gamma) \in V_{1} ; \\ \lambda_{n, 0}=-\mu_{n}^{-2 \alpha+\beta+1}(1+o(1)), \quad \lambda_{n, \pm}=-\frac{\mu_{n}^{\beta}}{2}(1+o(1)) \pm i \mu_{n}^{\alpha-\frac{\gamma}{2}}(1+o(1)), & (\alpha, \beta, \gamma) \in V_{2} ; \\ \lambda_{n, 0}=-\mu_{n}^{\beta}(1+o(1)), \quad \lambda_{n, \pm}=-\mu_{n}^{2 \alpha-\beta-\gamma}(1+o(1)), \quad \lambda_{n,-}=-\mu_{n}^{-2 \alpha+\beta+1}(1+o(1)), & (\alpha, \beta, \gamma) \in V_{3} \cup F_{3} ; \\ \lambda_{n, 0}=-\mu_{n}^{\beta}(1+o(1)), \quad \lambda_{n, \pm}=-\frac{1}{2} \mu_{n}^{2 \alpha-\beta-\gamma}(1+o(1)) \pm i \mu_{n}^{\frac{1}{2}-\frac{\gamma}{2}}(1+o(1)), & (\alpha, \beta, \gamma) \in V_{4} ; \\ \lambda_{n, 0}=-\frac{\mu_{n}^{\beta}}{2}(1+o(1)), \quad \lambda_{n, \pm}=-\frac{\mu_{n}^{\beta}}{4}(1+o(1)) \pm i \sqrt{2} \mu_{n}^{\frac{1}{2}-\frac{\gamma}{2}}(1+o(1)), & (\alpha, \beta, \gamma) \in F_{12} ; \\ \lambda_{n, 0}=-\mu_{n}^{\frac{1}{2}-\frac{\gamma}{2}}(1+o(1)), \quad \lambda_{n, \pm}=-\frac{1}{4} \mu_{n}^{2 \alpha-\frac{\gamma}{2}-\frac{1}{2}}(1+o(1)) \pm i \mu_{n}^{\frac{1}{2}-\frac{\gamma}{2}}(1+o(1)), & (\alpha, \beta, \gamma) \in F_{14} ; \\ \lambda_{n, 0}=-\mu_{n}^{-\alpha-\frac{\gamma}{2}+1}(1+o(1)), \quad \lambda_{n, \pm}=\left(-\frac{1}{2} \pm i \frac{\sqrt{3}}{2}\right) \mu_{n}^{\alpha-\frac{\gamma}{2}}(1+o(1)), & (\alpha, \beta, \gamma) \in F_{23} ; \\ \lambda_{n, 0}=-\mu_{n}^{\beta}(1+o(1)), \quad \lambda_{n, \pm}=\left(-\frac{1}{2} \pm i \frac{\sqrt{3}}{2}\right) \mu_{n}^{\frac{1}{2}-\frac{\gamma}{2}}(1+o(1)), & (\alpha, \beta, \gamma) \in F_{34} .\end{cases}
$$

- When $(\alpha, \beta, \gamma) \in L_{1234}$,

$$
\left\{\begin{array}{l}
\lambda_{n, 0}=\frac{1}{6}\left(2^{\frac{2}{3}}(3 \sqrt{69}-11)^{\frac{1}{3}}-2^{\frac{2}{3}}(3 \sqrt{69}+11)^{\frac{1}{3}}-2\right) \mu_{n}^{\frac{1}{2}-\frac{\gamma}{2}}(1+o(1)), \\
\lambda_{n,+}=\frac{1}{12}\left(2^{\frac{2}{3}}(-1+i \sqrt{3})(3 \sqrt{69}-11)^{\frac{1}{3}}+2^{\frac{2}{3}}(1+i \sqrt{3})(3 \sqrt{69}+11)^{\frac{1}{3}}-4\right) \mu_{n}^{\frac{1}{2}-\frac{\gamma}{2}}(1+o(1)), \\
\lambda_{n,-}=\frac{1}{12}\left(2^{\frac{2}{3}}(-1-i \sqrt{3})(3 \sqrt{69}-11)^{\frac{1}{3}}+2^{\frac{2}{3}}(1-i \sqrt{3})(3 \sqrt{69}+11)^{\frac{1}{3}}-4\right) \mu_{n}^{\frac{1}{2}-\frac{\gamma}{2}}(1+o(1)) .
\end{array}\right.
$$

Since $E \subseteq G$ is the parameter space of interest in this paper, we also summarize the leading terms of $\lambda_{n, 0}$ and $\lambda_{n, \pm}$ in various regions of $E$ in Corollary 5.5.

Corollary 5.5. Respectively in $R_{1}, R_{2}, R_{3}, R_{4}, S_{2}$, and $S_{3}$, the leading terms of $\lambda_{n, 0}$ and $\lambda_{n, \pm}$ are

$$
\left\{\begin{array}{lll}
\lambda_{n, 0}=-\mu_{n}^{\beta}, \quad \lambda_{n,+}=-\mu_{n}^{2 \alpha-\beta-\gamma}, \quad \lambda_{n,-}=-\mu_{n}^{-2 \alpha+\beta+1}, & (\alpha, \beta, \gamma) \in R_{1} \backslash\left(F_{23} \cup F_{34} \cup L_{1234}\right) ; \\
\lambda_{n, 0}=-\mu_{n}^{\beta}, \quad \lambda_{n, \pm}=-\frac{1}{2} \mu_{n}^{2 \alpha-\beta-\gamma} \pm i \mu_{n}^{\frac{1}{2}-\frac{\gamma}{2}}, & (\alpha, \beta, \gamma) \in R_{2} \backslash F_{14} ; \\
\lambda_{n, 0}=-\mu_{n}^{\beta}, \quad \lambda_{n, \pm}=-\frac{1}{2} \mu_{n}^{2 \alpha+\beta-1} \pm i \mu_{n}^{\frac{1}{2}-\frac{\gamma}{2}}, & (\alpha, \beta, \gamma) \in R_{3} ; \\
\lambda_{n, 0}=-\mu_{n}^{-2 \alpha+\beta+1}, \quad \lambda_{n, \pm}=-\frac{\mu_{n}^{\beta}}{2} \pm i \mu_{n}^{\alpha-\frac{\gamma}{2}}, & (\alpha, \beta, \gamma) \in R_{4} \backslash F_{12} ; \\
\lambda_{n, 0}=-\mu_{n}^{\beta}, \quad \lambda_{n, \pm}=-\frac{1}{2} \mu_{n}^{2 \alpha-\beta-\gamma} \pm i \mu_{n}^{\frac{1}{2}-\frac{\gamma}{2}}, & (\alpha, \beta, \gamma) \in S_{2} \backslash F_{14} ; \\
\lambda_{n, 0}=-\mu_{n}^{\beta}, \quad \lambda_{n, \pm}=-\frac{1}{2} \mu_{n}^{2 \alpha+\beta-1} \pm i \mu_{n}^{\frac{1}{2}-\frac{\gamma}{2}}, & (\alpha, \beta, \gamma) \in S_{3} .
\end{array}\right.
$$


Corollary 5.5 plays a crucial role: the decomposition of the parameter space $E$ into regions of polynomial stability, exponential stability, and regularity, as well as the order and the corresponding optimality of polynomial stability and Gevrey class, is all conjectured and derived based on the behavior of the leading terms of the roots of the characteristic equation reported in this corollary.

Subsequent sections will provide technical results leading to the proof of Theorem 5.4.

\subsection{Leading Terms of $A_{n}$ and $B_{n}$}

This section determines the leading terms of $A_{n}$ and $B_{n}$ as $n \rightarrow \infty$. Lemma 5.6 presents a method to compute these leading terms.

Lemma 5.6. If $-\frac{q_{n}}{2}=o\left(\sqrt{\Delta_{n}}\right)$ as $n \rightarrow \infty$, then

$$
\lim _{n \rightarrow \infty} A_{n}=\lim _{n \rightarrow \infty}-\frac{q_{n}}{p_{n}}=\lim _{n \rightarrow \infty}-\frac{1}{9 a_{n}} \cdot \frac{g_{n}}{h_{n}}, \quad \lim _{n \rightarrow \infty} B_{n}=\lim _{n \rightarrow \infty} \frac{6 \sqrt{\Delta_{n}}}{p_{n}}=\lim _{n \rightarrow \infty} \sqrt{3} \cdot \frac{\sqrt{W_{n}}}{h_{n}} .
$$

If $\sqrt{\Delta_{n}}=o\left(-\frac{q_{n}}{2}\right)$ as $n \rightarrow \infty$, then

$$
\lim _{n \rightarrow \infty} A_{n}=\lim _{n \rightarrow \infty} \frac{3 q_{n}}{p_{n}}=\lim _{n \rightarrow \infty} \frac{1}{3 a_{n}} \cdot \frac{g_{n}}{h_{n}}, \quad \lim _{n \rightarrow \infty} B_{n}=\lim _{n \rightarrow \infty}-\frac{2 \sqrt{\Delta_{n}}}{p_{n}}=\lim _{n \rightarrow \infty}-\frac{\sqrt{3}}{3} \cdot \frac{\sqrt{W_{n}}}{h_{n}} .
$$

If $\sqrt{\Delta_{n}}=O\left(-\frac{q_{n}}{2}\right)$ as $n \rightarrow \infty$, then

$$
\lim _{n \rightarrow \infty} A_{n}, B_{n}=\left(\left(\kappa_{1}+\kappa_{2}\right)^{\frac{1}{3}} \pm\left(\kappa_{1}-\kappa_{2}\right)^{\frac{1}{3}}\right) \mu_{n}^{x}(1+o(1)),
$$

where $\kappa_{1}, \kappa_{2}$, and $x$ are quantities related to $-\frac{q_{n}}{2}$ and $\sqrt{\Delta}$ via

$$
-\frac{q_{n}}{2}=\kappa_{1} \mu_{n}^{3 x}(1+o(1)), \quad \sqrt{\Delta_{n}}=\kappa_{2} \mu_{n}^{3 x}(1+o(1)) .
$$

In (5.16), $3 x$ is the power of the leading term shared by $-\frac{q_{n}}{2}$ and $\sqrt{\Delta_{n}}$ because we consider $\sqrt{\Delta_{n}}=O\left(-\frac{q_{n}}{2}\right)$. We further assume that $\kappa_{1} \pm \kappa_{2} \neq 0$. We omit the discussion when $\kappa_{1} \pm \kappa_{2}=0$ as it is not involved in our problem.

Proof. - If $-\frac{q_{n}}{2}=o\left(\sqrt{\Delta_{n}}\right)$, then by (5.8), $\Phi_{n, \pm}=O\left(\sqrt{\Delta_{n}}\right)$ and $\lim _{n \rightarrow \infty} \Phi_{n, \pm}=\lim _{n \rightarrow \infty} \pm \sqrt{\Delta_{n}} \Rightarrow$ $\lim _{n \rightarrow \infty} \Phi_{n,+}=-\lim _{n \rightarrow \infty} \Phi_{n,-} \Rightarrow \lim _{n \rightarrow \infty} \Phi_{n,+}^{\frac{1}{3}}=-\lim _{n \rightarrow \infty} \Phi_{n,-}^{\frac{1}{3}}$. Recall that $\Phi_{n,+}^{\frac{1}{3}} \Phi_{n,-}^{\frac{1}{3}}=-\frac{p_{n}}{3}$. We therefore have that $\lim _{n \rightarrow \infty} \Phi_{n, \pm}^{\frac{2}{3}}=\lim _{n \rightarrow \infty} \frac{p_{n}}{3}$. Therefore, by (5.9), we can show that (5.13) holds.

- If $\sqrt{\Delta_{n}}=o\left(-\frac{q_{n}}{2}\right)$, then by (5.8), $\Phi_{n, \pm}=O\left(-\frac{q_{n}}{2}\right)$ and $\lim _{n \rightarrow \infty} \Phi_{n, \pm}=\lim _{n \rightarrow \infty}-\frac{q_{n}}{2} \Rightarrow \lim _{n \rightarrow \infty} \Phi_{n,+}^{\frac{1}{3}}=$ $\lim _{n \rightarrow \infty} \Phi_{n,-}^{\frac{1}{3}}$. By $\Phi_{n,+}^{\frac{1}{3}} \Phi_{n,-}^{\frac{1}{3}}=-\frac{p_{n}}{3}$, we have that $\lim _{n \rightarrow \infty} \Phi_{n, \pm}^{\frac{2}{3}}=-\lim _{n \rightarrow \infty} \frac{p_{n}}{3}$. Therefore, by (5.9), we can show that (5.14) holds.

- Finally, when $\sqrt{\Delta_{n}}=O\left(-\frac{q_{n}}{2}\right)$, without loss of generality, we can write:

$$
-\frac{q_{n}}{2}=\kappa_{1} \mu_{n}^{3 x}(1+o(1)), \quad \sqrt{\Delta_{n}}=\kappa_{2} \mu_{n}^{3 x}(1+o(1)),
$$

where we further assume that $\kappa_{1} \pm \kappa_{2} \neq 0$. Then

$$
\Phi_{n, \pm}=\left(\kappa_{1} \pm \kappa_{2}\right) \mu_{n}^{3 x}(1+o(1)) \Rightarrow \Phi_{n, \pm}^{\frac{1}{3}}=\left(\kappa_{1} \pm \kappa_{2}\right)^{\frac{1}{3}} \mu_{n}^{x}(1+o(1)) .
$$

Therefore, by (5.9), (5.15) also holds. 
TABLE 1. Expression of $a_{n}, h_{n}, g_{n}$, and $W_{n}$ in various regions.

\begin{tabular}{ccrrr}
\hline Region & $a_{n}$ & \multicolumn{1}{c}{$h_{n}$} & \multicolumn{1}{c}{$g_{n}$} & \multicolumn{1}{c}{$W_{n}$} \\
\hline$V_{1}$ & $\mu_{n}^{\gamma}(1+o(1))$ & $3 \mu_{n}^{\gamma+1}(1+o(1))$ & $18 \mu_{n}^{\beta+2 \gamma+1}(1+o(1))$ & $4 \mu_{n}^{\gamma+3}(1+o(1))$ \\
$V_{2}$ & $\mu_{n}^{\gamma}(1+o(1))$ & $3 \mu_{n}^{2 \alpha+\gamma}(1+o(1))$ & $-9 \mu_{n}^{2 \alpha+\beta+2 \gamma}(1+o(1))$ & $4 \mu_{n}^{6 \alpha+\gamma}(1+o(1))$ \\
$V_{3} \cup F_{3}$ & $\mu_{n}^{\gamma}(1+o(1))$ & $-\mu_{n}^{2 \beta+2 \gamma}(1+o(1))$ & $2 \mu_{n}^{3 \beta+3 \gamma}(1+o(1))$ & $-\mu_{n}^{4 \alpha+2 \beta+2 \gamma}(1+o(1))$ \\
$V_{4}$ & $\mu_{n}^{\gamma}(1+o(1))$ & $-\mu_{n}^{2 \beta+2 \gamma}(1+o(1))$ & $2 \mu_{n}^{3 \beta+3 \gamma}(1+o(1))$ & $4 \mu_{n}^{4 \beta+3 \gamma+1}(1+o(1))$ \\
$F_{12}$ & $\mu_{n}^{\gamma}(1+o(1))$ & $6 \mu_{n}^{\gamma+1}(1+o(1))$ & $9 \mu_{n}^{\beta+2 \gamma+1}(1+o(1))$ & $32 \mu_{n}^{\gamma+3}(1+o(1))$ \\
$F_{14}$ & $\mu_{n}^{\gamma}(1+o(1))$ & $2 \mu_{n}^{\gamma+1}(1+o(1))$ & $20 \mu_{n}^{\frac{3 \gamma}{2}+\frac{3}{2}}(1+o(1))$ & $16 \mu_{n}^{\gamma+3}(1+o(1))$ \\
$F_{23}$ & $\mu_{n}^{\gamma}(1+o(1))$ & $2 \mu_{n}^{2 \alpha+\gamma}(1+o(1))$ & $-7 \mu_{n}^{3 \alpha+\frac{3 \gamma}{2}}(1+o(1))$ & $3 \mu_{n}^{6 \alpha+\gamma}(1+o(1))$ \\
$F_{34}$ & $\mu_{n}^{\gamma}(1+o(1))$ & $-\mu_{n}^{2 \beta+2 \gamma}(1+o(1))$ & $2 \mu_{n}^{3 \beta+3 \gamma}(1+o(1))$ & $3 \mu_{n}^{4 \beta+3 \gamma+1}(1+o(1))$ \\
$L_{1234}$ & $\mu_{n}^{\gamma}(1+o(1))$ & $5 \mu_{n}^{\gamma+1}(1+o(1))$ & $11 \mu_{n}^{\frac{3 \gamma}{2}+\frac{3}{2}}(1+o(1))$ & $23 \mu_{n}^{\gamma+3}(1+o(1))$ \\
\hline
\end{tabular}

Lemma 5.6 shows that the leading terms of $A_{n}$ and $B_{n}$ are given by various analytic expressions involving $h_{n}, g_{n}, a_{n}$, and $\sqrt{\Delta_{n}}$, depending on the relative order between $-\frac{q_{n}}{2}$ and $\sqrt{\Delta_{n}}$. Furthermore, from (5.6), (5.7), and (5.8), $-\frac{q_{n}}{2}$ and $\sqrt{\Delta_{n}}$ are also dependent on $g_{n}, W_{n}$, and $a_{n}$. Therefore, to determine the leading terms of $A_{n}$ and $B_{n}$, it suffices to determine the leading terms of $a_{n}, h_{n}, g_{n}$ and $W_{n}$. Without loss of generality, we show in detail how we can determine the leading term of $h_{n}$ in region $V_{1}$.

Note that from (5.4) and (5.6),

$$
h_{n}=3 \mu_{n}+3 \mu_{n}^{2 \alpha}+3 \mu_{n}^{2 \alpha+\gamma}-2 \mu_{n}^{2 \beta+\gamma}-\mu_{n}^{2 \beta+2 \gamma}-\mu_{n}^{2 \beta}+3 \mu_{n}^{\gamma+1} .
$$

The leading term of $h_{n}$ in region $V_{1}$ is the term in (5.17) with the largest exponent when $(\alpha, \beta, \gamma) \in V_{1}$. For each of the seven terms in (5.17), we assume in turn that the term in question is the leading term, and verify the assumption by solving a feasibility problem. For example, if we assume that $3 \mu_{n}$ is the leading term, define

$$
\tilde{V}_{1}=\left\{(\alpha, \beta, \gamma) \in V_{1} \mid 1>2 \alpha, 1>2 \alpha+\gamma, 1>2 \beta+\gamma, 1>2 \beta+2 \gamma, 1>2 \beta, 1>\gamma+1\right\}
$$

We need to verify the assumption by showing that in (5.18), $\tilde{V}_{1} \neq \emptyset$. Furthermore, if $V_{1}=\tilde{V}_{1}$, then $3 \mu_{n}$ is the unique leading term of $h_{n}$ in region $V_{1}$. In this way, the leading terms of $a_{n}, h_{n}, g_{n}$, and $W_{n}$ in various regions can be determined by solving a series of feasibility problems. In practice, such feasibility problems can be solved by the Reduce function in Mathematica.

The leading terms of $a_{n}, h_{n}, g_{n}$, and $W_{n}$ are summarized in Table 1 .

With the quantities in Table 1 , we are able to determine the leading terms of $-\frac{q_{n}}{2}$ and $\sqrt{\Delta_{n}}$ using (5.6), (5.7), and (5.8). Based on the leading terms of $-\frac{q_{n}}{2}$ and $\sqrt{\Delta_{n}}$, we can further determine their relative order in these regions as $n \rightarrow \infty$. The results are summarized in Table 2 .

As an illustrative example, we show how to compute $\sqrt{\Delta_{n}}$ in region $V_{1}$. From (5.7) and Table 1:

$$
\sqrt{\Delta_{n}}=\frac{\sqrt{W_{n}}}{6 \sqrt{3} a_{n}^{2}}=\frac{\sqrt{4 \mu_{n}^{\gamma+3}(1+o(1))}}{6 \sqrt{3}\left(\mu_{n}^{\gamma}(1+o(1))\right)^{2}}=\frac{2 \mu_{n}^{\frac{\gamma+3}{2}}(1+o(1))}{6 \sqrt{3} \mu_{n}^{2 \gamma}(1+o(1))}=\frac{\sqrt{3}}{9} \mu_{n}^{\frac{3}{2}-\frac{3 \gamma}{2}}(1+o(1)) .
$$

With Tables 1 and 2, the leading terms of $A_{n}$ and $B_{n}$ can be calculated according to Lemma 5.6. These leading terms are summarized in Table 3. 
TABLE 2. Expression of $-\frac{q_{n}}{2}, \sqrt{\Delta_{n}}$ and their relative order in various regions.

\begin{tabular}{cccc}
\hline Region & $-\frac{q_{n}}{2}$ & $\sqrt{\Delta_{n}}$ & Relative order \\
\hline$V_{1}$ & $-\frac{1}{3} \mu_{n}^{\beta-\gamma+1}(1+o(1))$ & $\frac{\sqrt{3}}{9} \mu_{n}^{\frac{3}{2}-\frac{3 \gamma}{2}}(1+o(1))$ & $-\frac{q_{n}}{2}=o\left(\sqrt{\Delta_{n}}\right)$ \\
$V_{2}$ & $\frac{1}{6} \mu_{n}^{2 \alpha+\beta-\gamma}(1+o(1))$ & $\frac{\sqrt{3}}{9} \mu_{n}^{3 \alpha-\frac{3 \gamma}{2}}(1+o(1))$ & $-\frac{q_{n}}{2}=o\left(\sqrt{\Delta_{n}}\right)$ \\
$V_{3} \cup F_{3}$ & $-\frac{1}{27} \mu_{n}^{3 \beta}(1+o(1))$ & $\frac{i \sqrt{3}}{18} \mu_{n}^{2 \alpha+\beta-\gamma}(1+o(1))$ & $\sqrt{\Delta_{n}}=o\left(-\frac{q_{n}}{2}\right)$ \\
$V_{4}$ & $-\frac{1}{27} \mu_{n}^{3 \beta}(1+o(1))$ & $\frac{\sqrt{3}}{9} \mu_{n}^{2 \beta-\frac{\gamma}{2}+\frac{1}{2}}(1+o(1))$ & $\sqrt{\Delta_{n}}=o\left(-\frac{q_{n}}{2}\right)$ \\
$F_{12}$ & $-\frac{1}{6} \mu_{n}^{\beta-\gamma+1}(1+o(1))$ & $\frac{2 \sqrt{6}}{9} \mu_{n}^{\frac{3}{2}-\frac{3 \gamma}{2}}(1+o(1))$ & $-\frac{q_{n}}{2}=o\left(\sqrt{\Delta_{n}}\right)$ \\
$F_{14}$ & $-\frac{10}{27} \mu_{n}^{\frac{3}{2}-\frac{3 \gamma}{2}}(1+o(1))$ & $\frac{2 \sqrt{3}}{9} \mu_{n}^{\frac{3}{2}-\frac{3 \gamma}{2}}(1+o(1))$ & $-\frac{q_{n}}{2}=o\left(\sqrt{\Delta_{n}}\right)$ \\
$F_{23}$ & $\frac{7}{54} \mu_{n}^{3 \alpha-\frac{3 \gamma}{2}}(1+o(1))$ & $\frac{1}{6} \mu_{n}^{3 \alpha-\frac{3 \gamma}{2}}(1+o(1))$ & $-\frac{q_{n}}{2}=o\left(\sqrt{\Delta_{n}}\right)$ \\
$F_{34}$ & $-\frac{1}{27} \mu_{n}^{3 \beta}(1+o(1))$ & $\frac{1}{6} \mu_{n}^{2 \beta-\frac{\gamma}{2}+\frac{1}{2}}(1+o(1))$ & $\sqrt{\Delta_{n}}=o\left(-\frac{q_{n}}{2}\right)$ \\
$L_{1234}$ & $-\frac{11}{54} \mu_{n}^{\frac{3}{2}-\frac{3 \gamma}{2}}(1+o(1))$ & $\frac{\sqrt{69}}{18} \mu_{n}^{\frac{3}{2}-\frac{3 \gamma}{2}}(1+o(1))$ & $-\frac{q_{n}}{2}=o\left(\sqrt{\Delta_{n}}\right)$ \\
\hline
\end{tabular}

TABLE 3. Expression of $A_{n}$ and $B_{n}$ in various regions.

\begin{tabular}{ccc}
\hline Region & $A_{n}$ & $B_{n}$ \\
\hline$V_{1}$ & $-\frac{2}{3} \mu_{n}^{\beta}(1+o(1))$ & $\frac{2 \sqrt{3}}{3} \mu_{n}^{\frac{1}{2}-\frac{\gamma}{2}}(1+o(1))$ \\
$V_{2}$ & $\frac{1}{3} \mu_{n}^{\beta}(1+o(1))$ & $\frac{2 \sqrt{3}}{3} \mu_{n}^{\alpha-\frac{\gamma}{2}}(1+o(1))$ \\
$V_{3} \cup F_{3}$ & $\frac{i \sqrt{3}}{3} \mu_{n}^{2 \alpha-\beta-\gamma}(1+o(1))$ \\
$V_{4}$ & $-\frac{2}{3} \mu_{n}^{\beta}(1+o(1))$ & $\frac{2 \sqrt{3}}{3} \mu_{n}^{\frac{1}{2}-\frac{\gamma}{2}}(1+o(1))$ \\
$F_{12}$ & $-\frac{2}{3} \mu_{n}^{\beta}(1+o(1))$ & $\frac{2 \sqrt{6}}{3} \mu_{n}^{\frac{1}{2}-\frac{\gamma}{2}}(1+o(1))$ \\
$F_{14}$ & $-\frac{1}{6} \mu_{n}^{\beta}(1+o(1))$ & $\frac{2 \sqrt{3}}{3} \mu_{n}^{\frac{1}{2}-\frac{\gamma}{2}}(1+o(1))$ \\
$F_{23}$ & $-\frac{2}{3} \mu_{n}^{\frac{1}{2}-\frac{\gamma}{2}}(1+o(1))$ & $\mu_{n}^{\alpha-\frac{\gamma}{2}}(1+o(1))$ \\
$F_{34}$ & $\frac{1}{3} \mu_{n}^{\alpha-\frac{\gamma}{2}}(1+o(1))$ & $\mu_{n}^{\frac{1}{2}}-\frac{\gamma}{2}(1+o(1))$ \\
$L_{1234}$ & $-\frac{2}{3} \mu_{n}^{\beta}(1+o(1))$ & $(\sqrt[3]{3 \sqrt{69}-11}+\sqrt[3]{11+3 \sqrt{69}})$ \\
\hline
\end{tabular}

\subsection{Lower order terms of $A_{n}$}

While Section 5.2 provides a characterization of the leading term of $A_{n}$, in subsequent derivation, we will show that just using the leading term of $A_{n}$ does not suffice to estimate the leading terms of $\lambda_{n, 0}$ and $\lambda_{n, \pm}$ as desired. This motivates us to further characterize the lower order terms of $A_{n}$, yielding Lemma 5.7.

Lemma 5.7. Let $A_{n}=Z_{n}+w_{n}$, where $Z_{n}$ is the leading term of $A_{n}$ as $n \rightarrow \infty$, and $w_{n}$ is the lower order term. i.e. $w_{n}=o\left(Z_{n}\right)$. Then

$$
\lim _{n \rightarrow \infty} w_{n}=\lim _{n \rightarrow \infty}-\frac{p_{n} Z_{n}+q_{n}+Z_{n}^{3}}{p_{n}+3 Z_{n}^{2}}=\lim _{n \rightarrow \infty}-\frac{27 a_{n}^{3} Z_{n}^{3}+9 a_{n} h_{n} Z_{n}+g_{n}}{81 a_{n}^{3} Z_{n}^{2}+9 a_{n} h_{n}} .
$$

Proof. We first define

$$
x_{n, 0}=A_{n}, \quad x_{n, \pm}=-\frac{1}{2} A_{n} \pm i \frac{\sqrt{3}}{2} B_{n} .
$$


By Cadano's formula, $x_{n, 0}, x_{n, \pm}$ are solutions to $x_{n}^{3}+p_{n} x_{n}+q_{n}=0$. We further define

$$
y_{n, 0}=w_{n}, \quad y_{n, \pm}=-\frac{w_{n}}{2}-\frac{3}{2} Z_{n} \pm i \frac{\sqrt{3}}{2} B_{n}
$$

where $A_{n}=Z_{n}+w_{n}, Z_{n}$ is the leading term of $A_{n}$, and $w_{n}=o\left(Z_{n}\right)$. Apparently,

$$
x_{n, 0}=Z_{n}+y_{n, 0}, \quad x_{n, \pm}=Z_{n}+y_{n, \pm}
$$

Therefore, $y_{n, 0}$ and $y_{n, \pm}$ are the three solutions for:

$$
\left(y_{n}+Z_{n}\right)^{3}+p_{n}\left(y+Z_{n}\right)+q_{n}=0 \Rightarrow y_{n}^{3}+3 Z_{n} y_{n}^{2}+\left(p_{n}+3 Z_{n}^{2}\right) y_{n}+p_{n} Z_{n}+q_{n}+Z_{n}^{3}=0 .
$$

On the other hand, using (5.20), by Vieta's formula with respect to $y_{n, 0}$, and $y_{n, \pm}$,

$$
\begin{gathered}
y_{n, 0} y_{n,+}+y_{n, 0} y_{n,-}+y_{n,+} y_{n,-}=\frac{3}{4} B_{n}^{2}+\frac{9}{4} Z_{n}^{2}-\frac{3}{2} Z_{n} w_{n}-\frac{3}{4} w_{n}^{2}=p_{n}+3 Z_{n}^{2}, \\
y_{n, 0}+y_{n,+}+y_{n,-}=\frac{3}{4} B_{n}^{2} w_{n}+\frac{9}{4} Z_{n}^{2} w_{n}+\frac{3}{2} Z_{n} w_{n}^{2}+\frac{1}{4} w_{n}^{3}=-\left(p_{n} Z_{n}+q_{n}+Z_{n}^{3}\right) .
\end{gathered}
$$

If $-\frac{q_{n}}{2}=o\left(\sqrt{\Delta_{n}}\right)$, by (5.13), $Z_{n}=o\left(B_{n}\right)$. From (5.21a) and (5.21b), as $n \rightarrow \infty$,

$$
\lim _{n \rightarrow \infty} \frac{3}{4} B_{n}^{2}=\lim _{n \rightarrow \infty} p_{n}+3 Z_{n}^{2}, \quad \lim _{n \rightarrow \infty} \frac{3}{4} B_{n}^{2} w_{n}=\lim _{n \rightarrow \infty}-\left(p_{n} Z_{n}+q_{n}+Z_{n}^{3}\right),
$$

which indicates that (5.19) is true.

If $\sqrt{\Delta_{n}}=o\left(-\frac{q_{n}}{2}\right)$, by (5.14), $B_{n}=o\left(Z_{n}\right)$. From (5.21a) and (5.21b), as $n \rightarrow \infty$,

$$
\lim _{n \rightarrow \infty} \frac{9}{4} Z_{n}^{2}=\lim _{n \rightarrow \infty} p_{n}+3 Z_{n}^{2}, \quad \lim _{n \rightarrow \infty} \frac{9}{4} Z_{n}^{2} w_{n}=\lim _{n \rightarrow \infty}-\left(p_{n} Z_{n}+q_{n}+Z_{n}^{3}\right),
$$

which indicates that (5.19) is also true in this case.

Finally, if $\sqrt{\Delta_{n}}=O\left(-\frac{q_{n}}{2}\right)$, by (5.15), $Z_{n}=O\left(B_{n}\right)$. From (5.21a) and (5.21b), as $n \rightarrow \infty$,

$$
\lim _{n \rightarrow \infty} \frac{3}{4} B_{n}^{2}+\frac{9}{4} Z_{n}^{2}=\lim _{n \rightarrow \infty} p_{n}+3 Z_{n}^{2}, \quad \lim _{n \rightarrow \infty} \frac{3}{4} B_{n}^{2} w_{n}+\frac{9}{4} Z_{n}^{2} w_{n}=\lim _{n \rightarrow \infty}-\left(p_{n} Z_{n}+q_{n}+Z_{n}^{3}\right),
$$

which validates (5.19) in this case.

Given the leading term of $A_{n}$, denoted as $Z_{n}$, Lemma 5.7 provides an analytic solution to the lower order term of $A_{n}$ to $Z_{n}$, denoted as $w_{n}$, via (5.19) as a function of $Z_{n}, a_{n}, h_{n}$, and $g_{n}$. For all the regions defined in Definition 5.3, $Z_{n}$ is given by Table 3 , and the leading terms of $a_{n}, h_{n}, g_{n}$ can be looked up via Table 1 . We summarize the expression of $w_{n}$ in Table 4 .

\subsection{Determine the leading terms of $\lambda_{n, 0}$ and $\lambda_{n, \pm}$}

With the results from Sections 5.2 and 5.3, we are now able to apply Cardano's Formula presented in Lemma 5.10 to compute the leading terms of $\lambda_{n, 0}$ and $\lambda_{n, \pm}$ in various regions. To proceed, we also need to 
TABLE 4. Expression of $w_{n}$ in various regions.

\begin{tabular}{cc}
\hline Region & $w_{n}$ \\
\hline$V_{1}$ & $\mu_{n}^{2 \alpha+\beta-1}(1+o(1))$ \\
$V_{2}$ & $-\mu_{n}^{-2 \alpha+\beta+1}(1+o(1))$ \\
$V_{3} \cup F_{3}$ & $\mu_{n}^{2 \alpha-\beta-\gamma}(1+o(1))$ \\
$V_{4}$ & $\mu_{n}^{2 \alpha-\beta-\gamma}(1+o(1))$ \\
$F_{12}$ & $-\frac{1}{16} \mu_{n}^{3 \beta+\gamma-1}(1+o(1))$ \\
$F_{14}$ & $\frac{1}{2} \mu_{n}^{2 \alpha-\frac{\gamma}{2}-\frac{1}{2}}(1+o(1))$ \\
$F_{23}$ & $-\mu_{n}^{-\alpha-\frac{\gamma}{2}+1}(1+o(1))$ \\
$F_{34}$ & $\mu_{n}^{\frac{1}{2}-\frac{\gamma}{2}}(1+o(1))$ \\
$L_{1234}$ & Not needed \\
&
\end{tabular}

TABLE 5. Intermediate quantities in (5.10).

\begin{tabular}{|c|c|c|}
\hline Region & $A_{n}$ & $-\frac{1}{2} A_{n}$ \\
\hline$V_{1}$ & $-\frac{2}{3} \mu_{n}^{\beta}+\mu_{n}^{2 \alpha+\beta-1}(1+o(1))$ & $\frac{1}{2} \mu_{n}^{\beta}-\frac{1}{2} \mu_{n}^{2 \alpha+\beta-1}(1+o(1))$ \\
\hline$V_{2}$ & $\frac{1}{3} \mu_{n}^{\beta}-\mu_{n}^{-2 \alpha+\beta+1}(1+o(1))$ & $-\frac{1}{6} \mu_{n}^{\beta}+\frac{1}{2} \mu_{n}^{-2 \alpha+\beta+1}(1+o(1))$ \\
\hline$V_{3} \cup F_{3}$ & $-\frac{2}{3} \mu_{n}^{\beta}+\mu_{n}^{2 \alpha-\beta-\gamma}(1+o(1))$ & $\frac{1}{3} \mu_{n}^{\beta}-\frac{1}{2} \mu_{n}^{2 \alpha-\beta-\gamma}(1+o(1))$ \\
\hline$V_{4}$ & $-\frac{2}{3} \mu_{n}^{\beta}+\mu_{n}^{2 \alpha-\beta-\gamma}(1+o(1))$ & $\frac{1}{3} \mu_{n}^{\beta}-\frac{1}{2} \mu_{n}^{2 \alpha-\beta-\gamma}(1+o(1))$ \\
\hline$F_{12}$ & $-\frac{1}{6} \mu_{n}^{\beta}-\frac{1}{16} \mu_{n}^{3 \beta+\gamma-1}(1+o(1))$ & $\frac{1}{12} \mu_{n}^{\beta}+\frac{1}{32} \mu_{n}^{3 \beta+\gamma-1}(1+o(1))$ \\
\hline$F_{14}$ & $-\frac{2}{3} \mu_{n}^{\frac{1}{2}-\frac{\gamma}{2}}+\frac{1}{2} \mu_{n}^{2 \alpha-\frac{\gamma}{2}-\frac{1}{2}}(1+o(1))$ & $\frac{1}{3} \mu_{n}^{\frac{1}{2}-\frac{\gamma}{2}}-\frac{1}{4} \mu_{n}^{2 \alpha-\frac{\gamma}{2}-\frac{1}{2}}(1+o(1))$ \\
\hline$F_{23}$ & $\frac{1}{3} \mu_{n}^{\alpha-\frac{\gamma}{2}}-\mu_{n}^{-\alpha-\frac{\gamma}{2}+1}(1+o(1))$ & $-\frac{1}{6} \mu_{n}^{\alpha-\frac{\gamma}{2}}+\frac{1}{2} \mu_{n}^{-\alpha-\frac{\gamma}{2}+1}(1+o(1))$ \\
\hline$F_{34}$ & $-\frac{2}{3} \mu_{n}^{\beta}+\mu_{n}^{\frac{1}{2}-\frac{\gamma}{2}}(1+o(1))$ & $\frac{\mu_{n}^{\beta}}{3}-\frac{1}{2} \mu_{n}^{\frac{1}{2}-\frac{\gamma}{2}}(1+o(1))$ \\
\hline$L_{1234}$ & $\sqrt[3]{3 \sqrt{69}-11}-\sqrt[3]{11+3 \sqrt{69}}) \mu_{n}^{\frac{1}{2}-\frac{\gamma}{2}}(1+o(1))$ & $(\sqrt[3]{3 \sqrt{69}-11}-\sqrt[3]{11+3 \sqrt{69}}) \mu^{\frac{1}{2}-\frac{\gamma}{2}}(1+o(1))$ \\
\hline & & \\
\hline Region & $\pm i \frac{\sqrt{3}}{2} B_{n}$ & $-\frac{b_{n}}{3 a_{n}}$ \\
\hline$V_{1}$ & $\pm i \mu_{n}^{\frac{1}{2}-\frac{\gamma}{2}}(1+o(1))$ & $-\frac{1}{3} \mu_{n}^{\beta}$ \\
\hline$V_{2}$ & $\pm i \mu_{n}^{\alpha-\frac{\gamma}{2}}(1+o(1))$ & $-\frac{1}{3} \mu_{n}^{\beta}$ \\
\hline$V_{3} \cup F_{3}$ & $\mp \frac{1}{2} \mu_{n}^{2 \alpha-\beta-\gamma}(1+o(1))$ & $-\frac{1}{3} \mu_{n}^{\beta}$ \\
\hline$V_{4}$ & $\pm i \mu_{n}^{\frac{1}{2}-\frac{\gamma}{2}}(1+o(1))$ & $-\frac{1}{3} \mu_{n}^{\beta}$ \\
\hline$F_{12}$ & $\pm i \sqrt{2} \mu_{n}^{\frac{1}{2}-\frac{\gamma}{2}}(1+o(1))$ & $-\frac{1}{3} \mu_{n}^{\beta}$ \\
\hline$F_{14}$ & $\pm i \mu_{n}^{\frac{1}{2}-\frac{\gamma}{2}}(1+o(1))$ & $-\frac{1}{3} \mu_{n}^{\frac{1}{2}-\frac{\gamma}{2}}$ \\
\hline$F_{23}$ & $\pm i \frac{\sqrt{3}}{2} \mu_{n}^{\alpha-\frac{\gamma}{2}}(1+o(1))$ & $-\frac{1}{3} \mu_{n}^{\alpha-\frac{\gamma}{2}}$ \\
\hline$F_{34}$ & $\pm i \frac{\sqrt{3}}{2} \mu_{n}^{\frac{1}{2}-\frac{\gamma}{2}}(1+o(1))$ & $-\frac{1}{3} \mu_{n}^{\beta}$ \\
\hline$L_{1234}$ & $\pm \frac{i(\sqrt[3]{3 \sqrt{69}-11}+\sqrt[3]{11+3 \sqrt{69}})}{2 \sqrt[3]{2} \sqrt{3}} \mu_{n}^{\frac{1}{2}-\frac{\gamma}{2}}(1+o(1))$ & $-\frac{1}{3} \mu_{n}^{\frac{1}{2}-\frac{\gamma}{2}}$ \\
\hline
\end{tabular}

notice that

$$
\frac{b_{n}}{3 a_{n}}=\frac{1}{3} \mu_{n}^{\beta} .
$$


Applying the results from Table 3, Table 4 and (5.22) to (5.10), we can compute the leading terms of $\lambda_{n, 0}$ and $\lambda_{n, \pm}$ given in Theorem 5.4. The intermediate quantities are summarized in Table 5.

Using Table 5, we can verify most of the results in Theorem 5.4. Furthermore, the necessity of computing the lower order terms of $A_{n}$ is evident as $-\frac{b_{n}}{3 a_{n}}$ could cancel out the leading terms in $A_{n}$ or $\frac{1}{2} A_{n}$. However, notice that in $V_{3} \cup F_{3}$,

$$
\lambda_{n, 0}=-\mu_{n}^{\beta}(1+o(1)), \quad \lambda_{n,+}=-\mu_{n}^{2 \alpha-\beta-\gamma}(1+o(1)), \quad \text { and } \quad \lambda_{n,-}=o\left(\mu_{n}^{2 \alpha-\beta-\gamma}\right),
$$

where we are not sure of the leading term of $\lambda_{n,-}$ due to cancellation. To identify the leading term of $\lambda_{n,-}$, consider the Vieta's formula

$$
\lim _{n \rightarrow \infty} \lambda_{n, 0} \lambda_{n,+} \lambda_{n,-}=\lim _{n \rightarrow \infty}-\frac{d_{n}}{a_{n}} \Rightarrow \lim _{n \rightarrow \infty} \mu_{n}^{2 \alpha-\gamma} \lambda_{n,-}=-\mu_{n}^{\beta-\gamma+1} \Rightarrow \lambda_{n,-}=-\mu_{n}^{-2 \alpha+\beta+1}(1+o(1))
$$

We therefore have concluded the determination of the leading terms of $\lambda_{n, 0}$ and $\lambda_{n, \pm}$ in various regions of the parameter space.

\section{Sharpness of THE ORDER OF GEVREy ClASS AND THE ORDER OF POLYNOMIAL STABILITY}

We now present the proof of Theorem 2.2, which shows that the orders of Gevrey class corresponding to (2.2) of Theorem 2.1 and the orders of polynomial stability corresponding to (1.6) are sharp.

Proof. From (3.8) and (3.14), we have that

$$
\varlimsup_{\lambda \in \mathbb{R},|\lambda| \rightarrow \infty}|\lambda|^{\mu(\alpha, \beta, \gamma)}\left\|(i \lambda-\mathcal{A})^{-1}\right\|<\infty, \quad \forall(\alpha, \beta, \gamma) \in R_{2} \cup R_{3} \cup R_{4} \cup S_{2} \cup S_{3} .
$$

where $\mu(\alpha, \beta, \gamma)$ takes value from (2.2) corresponding to the order of Gevrey class in $R_{2}, R_{3}$, and $R_{4}$ respectively; and $\mu(\alpha, \beta, \gamma)$ takes value from (1.6) corresponding to the order of polynomial stability in $S_{2}$ and $S_{3}$. Subsequently, it suffices to show that

$$
\varlimsup_{\lambda \in \mathbb{R},|\lambda| \rightarrow \infty}|\lambda|^{\mu(\alpha, \beta, \gamma)}\left\|(i \lambda-\mathcal{A})^{-1}\right\| \geq 2, \quad \forall(\alpha, \beta, \gamma) \in R_{2} \cup R_{3} \cup R_{4} \cup S_{2} \cup S_{3} .
$$

This is because through an argument of contradiction, if the orders corresponding to (2.2) and (1.6) are not sharp, then there exists $\varepsilon>0$ such that

$$
\varlimsup_{\lambda \in \mathbb{R},|\lambda| \rightarrow \infty}|\lambda|^{\mu(\alpha, \beta, \gamma)+\varepsilon}\left\|(i \lambda-\mathcal{A})^{-1}\right\|<\infty, \quad \forall(\alpha, \beta, \gamma) \in R_{2} \cup R_{3} \cup R_{4} \cup S_{2} \cup S_{3} .
$$

However, from (6.2), for $\varepsilon>0$,

$$
\varlimsup_{\lambda \in \mathbb{R},|\lambda| \rightarrow \infty}|\lambda|^{\mu(\alpha, \beta, \gamma)}\left\|(i \lambda-\mathcal{A})^{-1}\right\| \geq 2 \Rightarrow \varlimsup_{\lambda \in \mathbb{R},|\lambda| \rightarrow \infty}|\lambda|^{\mu(\alpha, \beta, \gamma)+\varepsilon}\left\|(i \lambda-\mathcal{A})^{-1}\right\| \rightarrow \infty,
$$

which contradicts against (6.3).

Therefore, in what follows, we show why (6.2) is true. To this end, notice that if $\lambda \in \mathbb{C}$, with $\operatorname{Re} \lambda \neq 0$, is an eigenvalue of $\mathcal{A}$ which is a densely defined closed operator on some Hilbert space $\mathcal{H}$ such that $(i \operatorname{Im} \lambda-\mathcal{A})^{-1}$ exists, then

$$
|\operatorname{Re} \lambda|\left\|(i \operatorname{Im} \lambda-\mathcal{A})^{-1}\right\| \geq 1
$$


Indeed, there exists an $x \in \mathcal{D}(\mathcal{A})$ with $\|x\|=1$ such that $\mathcal{A} x=(\mu+i \nu) x \Rightarrow \mu(i \nu-\mathcal{A})^{-1} x=-x$. Thus $(6.4)$ follows. Now, from Theorem 5.4, we know that $\mathcal{A}_{\alpha, \beta, \gamma}$ has a sequence of complex conjugate eigenvalues of the form:

$$
\lambda_{n, \pm}=-a \mu_{n}^{\xi}(1+o(1)) \pm i b \mu_{n}^{\eta}(1+o(1)),
$$

where $a>0, b>0, \eta>0$, and $\xi \in \mathbb{R}$. Let $\lambda=b \mu_{n}^{\eta}$ and with (6.4), we have

$$
\begin{aligned}
1 \leq a \mu_{n}^{\xi}(1+o(1))\left\|\left(i b \mu_{n}^{\eta}-\mathcal{A}_{\alpha, \beta, \gamma}\right)^{-1}\right\| & =\frac{a}{b^{\frac{\xi}{\eta}}}|\lambda|^{\frac{\xi}{\eta}}\left\|\left(i \lambda-\mathcal{A}_{\alpha, \beta, \gamma}\right)^{-1}\right\|(1+o(1)) \\
& \Rightarrow|\lambda|^{\frac{\xi}{\eta}}\left\|\left(i \lambda-\mathcal{A}_{\alpha, \beta, \gamma}\right)^{-1}\right\| \geq \frac{b^{\xi / \eta}}{a}(1+o(1)) .
\end{aligned}
$$

Now, we show that satisfying (6.5) in $R_{2}, R_{3}, R_{4}, S_{2}$, and $S_{3}$, respectively implies that (6.2) is also satisfied.

In region $R_{2}$ and $S_{2}$,

$$
\lambda_{n, \pm}= \begin{cases}-\frac{1}{2} \mu_{n}^{2 \alpha-\beta-\gamma}(1+o(1)) \pm i \mu_{n}^{\frac{1}{2}-\frac{\gamma}{2}}(1+o(1)), \quad \beta>\frac{1-\gamma}{2} \\ -\frac{1}{4} \mu_{n}^{2 \alpha-\frac{\gamma}{2}-\frac{1}{2}}(1+o(1)) \pm \mu_{n}^{\frac{1}{2}-\frac{\gamma}{2}}(1+o(1)), \quad \beta=\frac{1-\gamma}{2}\end{cases}
$$

Thus,

$$
\left\{\begin{array}{lll}
\frac{\xi}{\eta}=\frac{2 \alpha-\beta-\gamma}{(1-\gamma) / 2}, & \frac{b^{\xi / \eta}}{a}=2, & \beta>\frac{1-\gamma}{2} \\
\frac{\xi}{\eta}=\frac{2 \alpha-\frac{\gamma}{2}-\frac{1}{2}}{\frac{1}{2}-\frac{\gamma}{2}}, & \frac{b^{\xi / \eta}}{a}=4, & \beta=\frac{1-\gamma}{2}
\end{array}\right.
$$

which leads to

$$
|\lambda|^{\frac{2 \alpha-\beta-\gamma}{(1-\gamma) / 2}}\left\|\left(i \lambda-\mathcal{A}_{\alpha, \beta, \gamma}\right)^{-1}\right\| \geq 2(1+o(1)) .
$$

In region $R_{3}$ and $S_{3}$,

$$
\lambda_{n, \pm}=-\frac{1}{2} \mu_{n}^{2 \alpha+\beta-1}(1+o(1)) \pm i \mu_{n}^{\frac{1}{2}-\frac{\gamma}{2}}(1+o(1)) .
$$

Hence,

$$
\frac{\xi}{\eta}=\frac{2 \alpha+\beta-1}{(1-\gamma) / 2}, \quad \frac{b^{\xi / \eta}}{a}=2
$$

Consequently,

$$
|\lambda|^{\frac{2 \alpha+\beta-1}{(1-\gamma) / 2}}\left\|\left(i \lambda-\mathcal{A}_{\alpha, \beta, \gamma}\right)^{-1}\right\| \geq 2 .
$$

Finally, in $R_{4}$,

$$
\lambda_{n, \pm}= \begin{cases}-\frac{1}{2} \mu_{n}^{\beta}(1+o(1)) \pm i \mu_{n}^{\alpha-\frac{\gamma}{2}}(1+o(1)), & \alpha>\frac{1}{2} \\ -\frac{1}{4} \mu_{n}^{\beta}(1+o(1)) \pm i \sqrt{2} \mu_{n}^{\frac{1}{2}-\frac{\gamma}{2}}(1+o(1)), & \alpha=\frac{1}{2}\end{cases}
$$


Thus,

$$
\left\{\begin{array}{l}
\frac{\xi}{\eta}=\frac{\beta}{\alpha-\frac{\gamma}{2}}, \quad \frac{b^{\xi / \eta}}{a}=2, \quad \alpha>\frac{1}{2}, \\
\frac{\xi}{\eta}=\frac{\beta}{(1-\gamma) / 2}, \quad \frac{b^{\xi / \eta}}{a}=2^{\beta+2}, \quad \alpha=\frac{1}{2} .
\end{array}\right.
$$

and we again have

$$
|\lambda|^{\frac{\beta}{\alpha-\frac{\gamma}{2}}}\left\|\left(i \lambda-\mathcal{A}_{\alpha \beta, \gamma}\right)^{-1}\right\| \geq 2(1+o(1)) .
$$

Combining the above, we see that (6.2) is satisfied, which concludes our proof.

\section{REFERENCES}

[1] F. Ammar-Khodja, A. Bader and A. Benabdallah, Dynamic stabilization of systems via decoupling techniques. ESAIM: COCV 4 (1999) 577-593.

[2] A. Borichev and Y. Tomilov, Optimal polynomial decay of functions and operator semigroups. Math. Ann. 347 (2010) 455-478.

[3] G. Chen and D.L. Russell, A mathematical model for linear elastic system with structural damping. Quart. Appl. Math. 39 (1982) 433-454.

[4] S. Chen and R. Triggiani, Proof of extensions of two conjectures on structural damping for elastic systems. Pacific J. Math. 136 (1989) 15-55.

[5] S. Chen and R. Triggiani, Gevrey class semigroups arising from elastic systems with gentle dissipation: the case $0<\alpha<\frac{1}{2}$. Proc. AMS 110 (1990) 401-415.

[6] F. Dell'Oro, J.E. Muñoz Rivera and V. Pata, Stability properties of an abstract system with applications to linear thermoelastic plates. J. Evol. Equ. 13 (2013) 777-794.

[7] K.-J. Engel and R. Nagel, One-Parameter Semigroups for Linear Evolution Equations. Springer (2000).

[8] H.D. Fernández Sare, Z. Liu and R. Racke, Stability of abstract thermoelastic systems with inertial terms. J. Diff. Eqs. 267 (2019) 7085-7134.

[9] J. Hao and Z. Liu, Stability of an abstract system of coupled hyperbolic and parabolic equations. ZAMP 64 (2013) $1145-1159$.

[10] J. Hao, Z. Liu and J. Yong, Regularity analysis for an abstract system of coupled hyperbolic and parabolic equations. J. Diff. Eqs. 259 (2015) 4763-4798.

[11] I. Lasiecka and R. Triggiani, Analyticity, and lack thereof, of thermo-elastic semigroups. Control and partial differential equations. ESAIM Proc. 4 (1998).

[12] Z. Liu and M. Renardy, A note on the equations of a thermoelastic plate. Appl. Math. Lett. 8 (1995) 1-6.

[13] Z. Liu and J. Yong, Qualitative properties of certain $C_{0}$ semigroups arising in elastic systems with various dampings. Adv. Diff. Eqs. 3 (1998) 643-686.

[14] Z. Liu and S. Zheng, Semigroups Associated with Dissipative Systems. Chapman and Hall/CRC (1999).

[15] B.E. Meserve, Fundamental Concepts of Algebra. Addison-Wesley, Cambridge (1953).

[16] J.E. Muñoz Rivera and R. Racke, Large solutions and smoothing properties for nonlinear thermoelastic systems. J. Diff. Eqs. 127 (1996) 454-483.

[17] A. Pazy, Semigroups of Linear Operators and Applications to Partial Differential Equations. Springer-Verlag, New York (1983).

[18] D.L. Russell, A general framework for the study of indirect mechanisms in elastic systems. J. Math. Anal. Appl. 173 (1993) 339-358.

[19] S. Taylor, Chapter "Gevrey semigroups". Ph.D. thesis, School of Mathematics, University of Minnesota, 1989. 